\title{
One-Carbon Homologation of Primary Alcohols to Carboxylic Acids, Esters, and Amides via Mitsunobu Reactions with MAC Reagents
}

\author{
Natsuko Kagawa, ${ }^{* 1,2}$ Antoinette E. Nibbs, ${ }^{3}$ and Viresh H. Rawal ${ }^{* 3}$ \\ ${ }^{1}$ Graduate School of Pharmaceutical Sciences, Chiba University, 1-8-1 Inohana, Chiba 260-8675, Japan \\ ${ }^{2}$ Center for Environment, Health and Field Sciences, Chiba University, 6-2-1 Kashiwa-no-ha, Kashiwa \\ 277-0882, Japan \\ ${ }^{3}$ Department of Chemistry, University of Chicago, 5735 South Ellis Avenue, Chicago, Illinois 60637, \\ United States \\ knatsuko@faculty.chiba-u.jp and vrawal@uchicago.edu
}

\section{Table of Contents}

General Information

General Procedure for Mitsunobu Homologation Reaction ......................................... S-3

Mitsunobu Homologation Products ………………………..................................... S-3

General Procedure for Unmasking of the Mitsunobu Adducts....................................... S-15

Unmasked Esters, Amides, and Carboxylic Acids ………………………………........ -16

One-Pot Procedure for Conversion of Benzyl Alcohol to Homologated Amides ......... S-19

Selected NMR Spectra ................................................................................. 


\section{General Information}

Reactions were run in oven-dried glassware under a nitrogen atmosphere. Reactions were monitored by thin-layer chromatography (TLC) on EMD Millipore silica gel $60 \AA$ F254 plates or Merck silica gel $60 \mathrm{~F}_{254}$ plates, visualized by UV florescence quenching $(254 \mathrm{~nm})$, $\mathrm{I}_{2} / \mathrm{SiO}_{2}, \mathrm{PMA}$, Seebach's stain, or Hanessian's staining solution. Ambient temperature refers to $18-23{ }^{\circ} \mathrm{C}$. Lower temperatures were maintained using ice/water bath $\left(0{ }^{\circ} \mathrm{C}\right)$ or an EYELA PSL-2500A ( -40 to $\left.0{ }^{\circ} \mathrm{C}\right)$ bath. Flash column chromatography (EtOAc/Hexanes, $\mathrm{MeOH} / \mathrm{CH}_{2} \mathrm{Cl}_{2}$, or $\mathrm{MeOH} / \mathrm{CHCl}_{3}$ ) was performed on SiliCycle SiliaFlash $(40-63 \mu \mathrm{m})$ or Cica 60 (spherical/ 63-210 $\mu \mathrm{m}$ ) silica gel. Recycling preparative HPLC was performed on a JAI LC-9201 with a JAIGEL-1H and JAIGEL-2H GPC column (600 mm x $20 \mathrm{~mm}$ ), equipped with a guard column and employing chloroform. NMR spectra were measured on Bruker DRX, DMX, or SMP spectrometers at $500 \mathrm{MHz}$ for ${ }^{1} \mathrm{H}$ spectra and $125 \mathrm{MHz}$ for ${ }^{13} \mathrm{C}$ spectra, or JEOL JNM-ECA600 at 600 $\mathrm{MHz}$ for ${ }^{1} \mathrm{H}$ spectra and $150 \mathrm{MHz}$ for ${ }^{13} \mathrm{C}$ spectra. ${ }^{1} \mathrm{H}$ spectra were calibrated from internal standard TMS $(\delta 0.0)$ or solvent resonance $\left(\mathrm{CHCl}_{3}: 7.26\right) .{ }^{13} \mathrm{C}$ spectra were calibrated from solvent resonance $\left(\mathrm{CHCl}_{3}: 77.0\right)$. NMR data are reported as: chemical shift (parts per million, ppm), multiplicity $(\mathrm{s}=$ singlet, $\mathrm{d}=$ doublet, $\mathrm{t}=$ triplet, $\mathrm{q}=$ quartet, $\mathrm{m}=$ multiplet, $\mathrm{br}=$ broad signal), coupling constant $(\mathrm{Hz})$, and integration. Infrared spectra were recorded on a Jasco FT/IR-4700 spectrometer with Smiths DuraSamplIR II (ATR) and reported in frequency of absorption $\left(\mathrm{cm}^{-1}\right)$. High-resolution mass spectral analyses using electrospray ionization (ESI) were measured on an Agilent Technologies 6224 TOF LC/MS or a JEOL JMS-T100 AccuTOF LC-plus. High-resolution mass spectra using fast atom bombardment (FAB) or electron ionization (EI) were reported at Kyoto Institute of Technology, Kyoto, on a JEOL JMS-700 Mstation, or at JEOL Ltd., Tokyo, on a JEOL JMS-700V. Gas chromatography mass spectral analysis was measured on a Varian Saturn 2200 GC-MS-MS. Optical rotations were measured on a Jasco P-2200 polarimeter using $50 \mathrm{~mm}$ path-length cell.

\section{Materials}

Tetrahydrofuran (THF) and methylene chloride $\left(\mathrm{CH}_{2} \mathrm{Cl}_{2}\right)$ were purified by passage over activated alumina, using an Innovative Technology, Inc. Puresolv solvent purification system. Anhydrous THF was also purchased from Wako Pure Chemical Industries, Ltd. Anhydrous $\mathrm{MeOH}, \mathrm{DME}$ and $\mathrm{CH}_{2} \mathrm{Cl}_{2}$ were purchased from Kanto Chemical Co., Inc. All other solvents were purchased from Fisher Scientific and used as received. All commercially obtained reagents were used as received. Substrate alcohols were acquired commercially. DIAD, DMEAD and $\mathrm{PPh}_{3}$ were purchased from Sigma-Aldrich and Tokyo Chemical Industry Co., Ltd. MOM-MAC 2a was synthesized according to previously published procedures. ${ }^{1}$ Ac-MAC $\mathbf{2 b}$ was both synthesized ${ }^{2}$ and commercially available

\footnotetext{
${ }^{1}$ Yang, K. S.; Nibbs, A. E.; Türkmen, Y. E.; Rawal, V. H. J. Am. Chem. Soc. 2013, 135, 16050-16053

${ }^{2}$ Nemoto, H.; Li, X.; Ma, R.; Suzuki, I.; Shibuya, M. Tetrahedron. Lett. 2003, 44, 73-75.
} 
from Masuda Chemical Industries Co., Ltd. (Z)-2-iodobut-2-en-1-ol ${ }^{3}$ and 2-(3',3'dimethyl-6-nitrospiro[chromene-2,2'-indolin]-1'-yl)ethan-1-ol ${ }^{4}$ were prepared according to literature procedure.

\section{General Procedure for Mitsunobu Homologation Reaction}

To an oven-dried two-neck round bottom flask was added a stir bar, $\mathrm{PPh}_{3}(115 \mathrm{mg}, 0.44$ mmol), and solvent ( $2 \mathrm{~mL})$. The flask was capped with a septum and connected to a $\mathrm{N}_{2}$ atmosphere. The mixture was cooled with an ice/water bath, and DIAD (0.44 mmol) was added. The mixture was allowed to stir for $30 \mathrm{~min}$, then $\mathrm{ROH}(0.44 \mathrm{mmol})$ was added in one portion. After an additional $30 \mathrm{~min}$, the MAC reagent $(50 \mathrm{mg}, 0.4 \mathrm{mmol}$ ) was added. The ice/water bath was allowed to warm to ambient temperature, and the reaction was monitored by TLC. After the MAC reagent was consumed, the reaction mixture was concentrated in vacuo. The residue was purified by flash column chromatography to afford the desired homologated product.

\section{Mitsunobu Homologation Products}

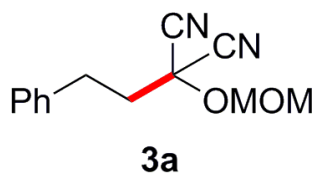

2-(Methoxymethoxy)-2-phenethylmalononitrile (3a): Prepared according to the general procedure using $\mathrm{PPh}_{3}(115 \mathrm{mg}, 0.44 \mathrm{mmol})$, THF (1 mL), DIAD (87 $\left.\mu \mathrm{L}, 0.44 \mathrm{mmol}\right)$, phenethyl alcohol $(53 \mu \mathrm{L}, 0.44 \mathrm{mmol})$, and MOM-MAC (50 mg, $0.4 \mathrm{mmol})$ for $24 \mathrm{~h}$. Purification by flash column chromatography ( $\mathrm{SiO}_{2}, 15 \%$ EtOAc/Hexanes) afforded 3a (75 mg, 82\%) as a pale yellow (almost-colorless) oil.

Analytical data for 3a:

${ }^{1} \mathbf{H}$ NMR $\left(600 \mathrm{MHz}, \mathrm{CDCl}_{3}\right): \delta 7.33(\mathrm{t}, J=7.8 \mathrm{~Hz}, 2 \mathrm{H}), 7.26(\mathrm{t}, J=7.8 \mathrm{~Hz}, 1 \mathrm{H}), 7.22(\mathrm{~d}$, $J=7.8 \mathrm{~Hz}, 2 \mathrm{H}), 5.07(\mathrm{~s}, 2 \mathrm{H}), 3.56(\mathrm{~s}, 3 \mathrm{H}), 2.99-2.96(\mathrm{~m}, 2 \mathrm{H}), 2.52-2.49(\mathrm{~m}, 2 \mathrm{H})$

${ }^{13}$ C NMR (150 MHz, $\left.\mathrm{CDCl}_{3}\right): \delta 138.1,128.8(\mathrm{x} 2), 128.4(\mathrm{x} 2), 126.9,113.1(\mathrm{x} 2), 96.2$, $65.3,57.4,41.7,29.8$

IR (ATR): 1455, 1164, 1090, 1031, 985, 925, 754, 699

HRMS (FAB) calcd for $\left(\mathrm{C}_{13} \mathrm{H}_{14} \mathrm{O}_{2} \mathrm{~N}_{2}\right)^{+}[\mathrm{M}]^{+}: 230.1055$. Found: 230.1061

\footnotetext{
${ }^{3}$ Wanner, M. J.; Boots, R. N. A.; Eradus, B.; de Gelder, R.; van Maarseveen, J. H.; Hiemstra, H. Org. Lett. 2009, 11, 2579-2581.

${ }^{4}$ Raymo, F. M.; Giordani, S. J. Org. Chem. 2003, 68, 4158-4169.
} 
<smiles>COC(C#N)(C#N)Cc1ccccc1</smiles>

$3 b$

2-Benzyl-2-(methoxymethoxy)malononitrile (3b): Prepared according to the general procedure using $\mathrm{PPh}_{3}(164 \mathrm{mg}, 0.63 \mathrm{mmol})$, THF (2.5 mL), DIAD (123 $\left.\mu \mathrm{L}, 0.63 \mathrm{mmol}\right)$, benzyl alcohol $(65 \mu \mathrm{L}, 0.63 \mathrm{mmol})$, and MOM-MAC $(52.7 \mathrm{mg}, 0.42 \mathrm{mmol})$ for $43 \mathrm{~h}$. Purification by flash column chromatography $\left(\mathrm{SiO}_{2}, 10 \%\right.$ EtOAc/Hexanes) afforded $\mathbf{3 b}$ (81.2 $\mathrm{mg}, 90 \%)$ as a colorless oil.

Analytical data for 3b:

${ }^{1} \mathbf{H}$ NMR (500 MHz, $\left.\mathrm{CDCl}_{3}\right): \delta 7.39$ (s, 5H), 5.03 (s, 2H), $3.48(\mathrm{~s}, 3 \mathrm{H}), 3.46$ (s, 2H)

${ }^{13}$ C NMR (125 MHz, $\left.\mathrm{CDCl}_{3}\right): \delta 130.8$ (x2), 130.2, 128.8, 128.7 (x2), 112.9 (x2), 96.2, $66.5,57.2,45.7$

IR (ATR): 1456, 1162, 1071, 1017, 969, 921, 767, 700

HRMS (FAB) calcd for $\left(\mathrm{C}_{12} \mathrm{H}_{12} \mathrm{O}_{2} \mathrm{~N}_{2} \mathrm{Na}\right)^{+}[\mathrm{M}+\mathrm{Na}]^{+}:$239.0796. Found: 239.0802

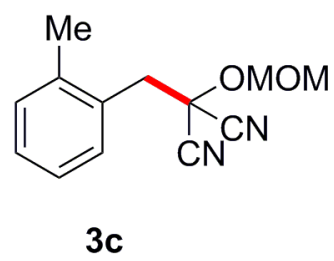

2-(Methoxymethoxy)-2-(2-methylbenzyl)malononitrile (3c): Prepared according to the general procedure using $\mathrm{PPh}_{3}(194 \mathrm{mg}, 0.74 \mathrm{mmol})$, THF (3.5 mL), DIAD (146 $\mu \mathrm{L}, 0.74$ mmol), 2-methylbenzyl alcohol (91 mg, $0.74 \mathrm{mmol})$, and MOM-MAC (62.2 $\mathrm{mg}, 0.49$ mmol) for $41 \mathrm{~h}$. Purification by flash column chromatography $\left(\mathrm{SiO}_{2} \quad 10 \%\right.$ EtOAc/Hexanes) afforded 3c (97.5 mg, 86\%) as a colorless oil.

Analytical data for 3c:

${ }^{1} \mathbf{H}$ NMR $\left(600 \mathrm{MHz}, \mathrm{CDCl}_{3}\right): \delta 7.39(\mathrm{~d}, J=7.2 \mathrm{~Hz}, 1 \mathrm{H}), 7.28-7.20(\mathrm{~m}, 3 \mathrm{H}) 5.01$ (s, $2 \mathrm{H}), 3.54(\mathrm{~s}, 2 \mathrm{H}), 3.48(\mathrm{~s}, 3 \mathrm{H}), 244(\mathrm{~s}, 3 \mathrm{H})$

${ }^{13} \mathbf{C}$ NMR $\left(150 \mathrm{MHz}, \mathrm{CDCl}_{3}\right): \delta 138.1,131.6,131.0,128.9,128.8,126.2,113.1$ (x2), $96.2,66.3,57.3,42.2,20.0$

IR (ATR): 1444, 1163, 1069, 1014, 968, 774, 748, 728

HRMS (FAB) calcd for $\left(\mathrm{C}_{13} \mathrm{H}_{14} \mathrm{O}_{2} \mathrm{~N}_{2} \mathrm{Na}\right)^{+}[\mathrm{M}+\mathrm{Na}]^{+}:$253.0953. Found: 253.0961 


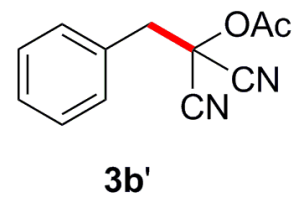

1,1-Dicyano-2-phenylethyl acetate (3b'): Prepared according to the general procedure using $\mathrm{PPh}_{3}(115 \mathrm{mg}, 0.44 \mathrm{mmol})$, THF $(1 \mathrm{~mL})$, DIAD $(87 \mu \mathrm{L}, 0.44 \mathrm{mmol})$, benzyl alcohol (46 $\mu \mathrm{L}, 0.44 \mathrm{mmol}$ ), and MOM-MAC (49 mg, $0.4 \mathrm{mmol}$ ) for $48 \mathrm{~h}$. Purification by flash column chromatography $\left(\mathrm{SiO}_{2}, 25 \%\right.$ EtOAc/Hexanes) and recycling preparative HPLC afforded 3b' (58 mg, 68\%) as a colorless oil.

Analytical data for $3 \mathbf{b}^{\prime}$ :

${ }^{1} \mathbf{H}$ NMR $\left(600 \mathrm{MHz} ; \mathrm{CDCl}_{3}\right): \delta 7.416(\mathrm{~s}, 5 \mathrm{H}), 3.552(\mathrm{~s}, 2 \mathrm{H}), 2.229(\mathrm{~s}, 3 \mathrm{H})$

${ }^{13}$ C NMR (150 MHz; $\left.\mathrm{CDCl}_{3}\right): \delta$ 169.4, $130.9(\mathrm{x} 2), 129.24,129.21,128.9(\mathrm{x} 2), 111.6$ (x2), 62.3, 43.9, 20.0

IR (ATR): 1773, 1371, 1197, 1034, 766, 701

HRMS (FAB) calcd for $\left(\mathrm{C}_{12} \mathrm{H}_{11} \mathrm{O}_{2} \mathrm{~N}_{2}\right)[\mathrm{M}+\mathrm{H}]^{+}: 215.0820$. Found: 215.0816

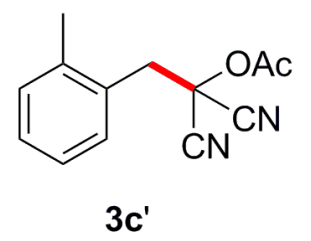

1,1-Dicyano-2-(o-tolyl)ethyl acetate (3c'): Prepared according to the general procedure using $\mathrm{PPh}_{3}(115 \mathrm{mg}, 0.44 \mathrm{mmol})$, THF $(1 \mathrm{~mL})$, DIAD $(87 \mu \mathrm{L}, 0.44 \mathrm{mmol}), 2$ methylbenzyl alcohol (54 mg, $0.44 \mathrm{mmol}$ ), and MOM-MAC (49 mg, $0.4 \mathrm{mmol})$ for $48 \mathrm{~h}$. Purification by flash column chromatography $\left(\mathrm{SiO}_{2}, 25 \%\right.$ EtOAc/Hexanes) and recycling preparative HPLC afforded 3c' (71 mg, 78\%) as a colorless oil.

Analytical data for 3c':

${ }^{1} \mathbf{H}$ NMR $\left(600 \mathrm{MHz} ; \mathrm{CDCl}_{3}\right): \delta 7.41(\mathrm{~d}, J=7.2,1 \mathrm{H}), 7.29(\mathrm{td}, J=7.2 \mathrm{~Hz}, 1.8 \mathrm{~Hz}, 1 \mathrm{H})$, $7.26(\mathrm{~d}, J=7.8 \mathrm{~Hz}, 1 \mathrm{H}), 7.24(\mathrm{t}, J=7.2 \mathrm{~Hz}, 1 \mathrm{H}), 3.63(\mathrm{~s}, 2 \mathrm{H}), 2.46(\mathrm{~s}, 3 \mathrm{H}), 2.22(\mathrm{~s}, 3 \mathrm{H})$

${ }^{13}$ C NMR (150 MHz; $\left.\mathrm{CDCl}_{3}\right): \delta 167.3,138.1,131.6,131.2,129.2,127.9,126.4,111.8$ (x2), 62.0, 40.4, 20.1, 19.9

IR (ATR): 1775, 1371, 1195, 1091, 1031, 925, 854, 775, 748, 723, 664

HRMS (FAB) calcd for $\left(\mathrm{C}_{13} \mathrm{H}_{13} \mathrm{O}_{2} \mathrm{~N}_{2}\right)[\mathrm{M}+\mathrm{H}]^{+}:$229.0977. Found: 229.0987 


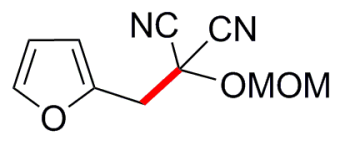

$3 d$

2-(Furan-2-ylmethyl)-2-(methoxymethoxy)malononitrile (3d): Prepared according to the general procedure using $\mathrm{PPh}_{3}(157 \mathrm{mg}, 0.60 \mathrm{mmol})$, THF $(2.5 \mathrm{~mL})$, DIAD $(118 \mu \mathrm{L}$, $0.60 \mathrm{mmol})$, furfuryl alcohol $(52 \mu \mathrm{L}, 0.60 \mathrm{mmol})$, and MOM-MAC (50.4 mg, $0.40 \mathrm{mmol})$ for $8 \mathrm{~h}$. Purification by flash column chromatography $\left(\mathrm{SiO}_{2}, 14 \%\right.$ EtOAc/Hexanes) afforded $3 \mathbf{d}$ (58 $\mathrm{mg}, 70 \%)$ as a colorless oil.

Analytical data for 3d:

${ }^{1} \mathbf{H}$ NMR $\left(500 \mathrm{MHz}, \mathrm{CDCl}_{3}\right): \delta 7.46(\mathrm{dd}, J=1.9,0.9 \mathrm{~Hz}, 1 \mathrm{H}), 6.48(\mathrm{~d}, J=3.3 \mathrm{~Hz}, 1 \mathrm{H})$, $6.41(\mathrm{dd}, J=3.2,1.8 \mathrm{~Hz}, 1 \mathrm{H}), 5.05(\mathrm{~s}, 3 \mathrm{H}), 3.57(\mathrm{~s}, 3 \mathrm{H}), 3.52(\mathrm{~s}, 3 \mathrm{H})$

${ }^{13}$ C NMR (125 MHz, $\left.\mathrm{CDCl}_{3}\right): \delta 144.4,143.7,112.6(\mathrm{x} 2), 111.3,110.9,96.3,65.1,57.3$, 38.9

IR (ATR): 1203, 1163, 1073, 1025, 967, 921, 748

HRMS (FAB) calcd for $\left(\mathrm{C}_{10} \mathrm{H}_{10} \mathrm{O}_{3} \mathrm{~N}_{2}\right) \mathrm{Na}^{+}[\mathrm{M}+\mathrm{Na}]^{+}: 229.0589$. Found: 229.0587

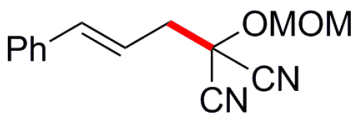

$3 e$

2-Cinnamyl-2-(methoxymethoxy)malononitrile (3e): Prepared according to the general procedure using $\mathrm{PPh}_{3}(157 \mathrm{mg}, 0.60 \mathrm{mmol})$, THF $(2.5 \mathrm{~mL})$, DIAD $(118 \mu \mathrm{L}, 0.60 \mathrm{mmol})$, cinnamyl alcohol $(87.0 \mathrm{mg}, 0.65 \mathrm{mmol})$, and MOM-MAC $(50.4 \mathrm{mg}, 0.40 \mathrm{mmol})$ for $4 \mathrm{~h}$. Purification by flash column chromatography $\left(\mathrm{SiO}_{2}, 14 \%\right.$ EtOAc/Hexanes) afforded $3 \mathbf{e}$ (80.9 $\mathrm{mg}, 84 \%)$ as a pale yellow (almost-colorless) oil.

Analytical data for 3e:

${ }^{1} \mathbf{H}$ NMR $\left(600 \mathrm{MHz}, \mathrm{CDCl}_{3}\right): \delta 7.41(\mathrm{dd}, J=7.2,1.8 \mathrm{~Hz}, 2 \mathrm{H}), 7.34(\mathrm{td}, J=7.2,1.8 \mathrm{~Hz}$, $2 \mathrm{H}), 7.29$ (tt, $J=7.8,1.8 \mathrm{~Hz}, 1 \mathrm{H}), 6.73(\mathrm{~d}, J=16 \mathrm{~Hz}, 1 \mathrm{H}), 6.19(\mathrm{dt}, J=16,7.2 \mathrm{~Hz}, 1 \mathrm{H})$, $5.05(\mathrm{~s}, 2 \mathrm{H}), 3.54(\mathrm{~s}, 3 \mathrm{H}), 3.08(\mathrm{dd}, J=7.8,1.8 \mathrm{~Hz}, 2 \mathrm{H})$

${ }^{13}$ C NMR (150 MHz, $\left.\mathrm{CDCl}_{3}\right): \delta 138.5,135.7,128.6$ (x2), 128.4, 126.7 (x2), 117.3, 113.0 (x2), 96.3, 65.8, 57.3, 43.5

IR (ATR): 1449, 1163, 1072, 1032, 965, 749, 694

HRMS (FAB) calcd for $\left(\mathrm{C}_{14} \mathrm{H}_{14} \mathrm{O}_{2} \mathrm{~N}_{2}\right)^{+}[\mathrm{M}]^{+}:$242.1055. Found: 242.1055 


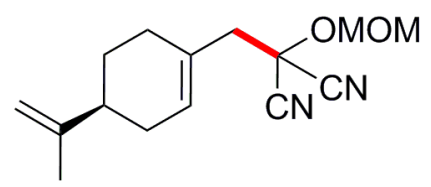

$3 f$

(S)-2-(Methoxymethoxy)-2-((4-(prop-1-en-2-yl)cyclohex-1-en-1-

yl)methyl)malononitrile (3f): Prepared according to the general procedure using $\mathrm{PPh}_{3}$ (115 mg, $0.44 \mathrm{mmol})$, THF (1 mL), DIAD (87 $\mu \mathrm{L}, 0.44 \mathrm{mmol}),(s)-(-)$-perillyl alcohol $(70 \mu \mathrm{L}, 0.44 \mathrm{mmol})$, and MOM-MAC (43 mg, $0.34 \mathrm{mmol}$ ) for $18 \mathrm{~h}$. Purification by flash column chromatography $\left(\mathrm{SiO}_{2}, 9 \%\right.$ EtOAc/Hexanes) afforded $\mathbf{3 f}(71 \mathrm{mg}, 81 \%)$ as a colorless oil.

Analytical data for 3f:

${ }^{1} \mathbf{H}$ NMR (600 MHz; $\left.\mathrm{CDCl}_{3}\right): \delta 5.90(\mathrm{~d}, J=2.4 \mathrm{~Hz}, 1 \mathrm{H}), 5.04(\mathrm{~s}, 2 \mathrm{H}), 4.73(\mathrm{dt}, J=16,1.5$ $\mathrm{Hz}, 2 \mathrm{H}), 3.54$ (s, 3H), 2.83 (s, 2H), $2.30-2.14$ (m, 4H), $2.07-1.99$ (m, 1H), 1.84 (dtd, J $=13,6.0,3.0 \mathrm{~Hz}, 1 \mathrm{H}), 1.74(\mathrm{~s}, 3 \mathrm{H}), 1.51(\mathrm{dtd}, J=13,11,5.4 \mathrm{~Hz}, 1 \mathrm{H})$

${ }^{13} \mathbf{C}$ NMR (150 MHz; $\left.\mathrm{CDCl}_{3}\right): \delta 149.0,130.9,128.2,113.3,113.2,108.9,96.1,66.2$, $57.2,47.4,40.1,30.8,29.9,27.5,20.7$

IR (ATR): 2921, 1437, 1164, 1069, 1025, 981, 937, 890

HRMS (FAB) calcd for $\left(\mathrm{C}_{15} \mathrm{H}_{21} \mathrm{O}_{2} \mathrm{~N}_{2}\right)[\mathrm{M}+\mathrm{H}]^{+}:$261.1603. Found: 261.1598

$[\alpha]^{23.8}=-58.38^{\circ}\left(\mathrm{c} 1.0, \mathrm{CHCl}_{3}\right)$

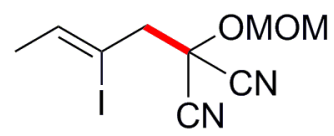

$3 g$

(Z)-2-(2-Iodobut-2-en-1-yl)-2-(methoxymethoxy)malononitrile (3g): Prepared according to the general procedure using $\mathrm{PPh}_{3}(149 \mathrm{mg}, 0.57 \mathrm{mmol})$, THF (2 mL), DIAD (112 $\mu \mathrm{L}, 0.57 \mathrm{mmol}),(Z)-2$-iodobut-2-en-1-ol (112 $\mathrm{mg}, 0.57 \mathrm{mmol})$, and MOM-MAC (48 mg, $0.38 \mathrm{mmol}$ ) for $17 \mathrm{~h}$. Purification by flash column chromatography $\left(\mathrm{SiO}_{2}, 5 \%\right.$ EtOAc/Hexanes) afforded $\mathbf{3 g}$ (93 mg, 80\%) as a colorless oil.

Analytical data for 3g:

${ }^{1} \mathbf{H}$ NMR $\left(600 \mathrm{MHz} ; \mathrm{CDCl}_{3}\right): \delta 6.10(\mathrm{q}, J=6.0 \mathrm{~Hz}, 1 \mathrm{H}), 5.06(\mathrm{~s}, 2 \mathrm{H}), 3.55(\mathrm{~s}, 3 \mathrm{H}), 3.42$ $(\mathrm{s}, 2 \mathrm{H}), 1.85(\mathrm{~d}, J=6.0 \mathrm{~Hz}, 3 \mathrm{H})$

${ }^{13}$ C NMR (150 MHz; $\left.\mathrm{CDCl}_{3}\right): \delta$ 141.0, 112.5 (x2), 96.1, 90.8, 65.8, 57.4, 52.6, 22.9

IR (ATR):1215, 1163, 1026, 971, 919, 757, 690, 609 
HRMS (ESI) calcd for $\left(\mathrm{C}_{9} \mathrm{H}_{11} \mathrm{O}_{2} \mathrm{~N}_{2} \mathrm{I}_{1} \mathrm{Na}_{1}\right)[\mathrm{M}+\mathrm{Na}]^{+}:$328.9763. Found: 328.9754

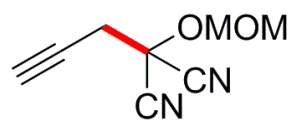

$3 \mathrm{~h}$

2-(Methoxymethoxy)-2-(prop-2-yn-1-yl)malononitrile (3h): Prepared according to the general procedure using $\mathrm{PPh}_{3}(164 \mathrm{mg}, 0.625 \mathrm{mmol})$, THF $(3 \mathrm{~mL})$, DIAD $(123 \mu \mathrm{L}, 0.625$ mmol), propargyl alcohol ( $36 \mu \mathrm{L}, 0.63 \mathrm{mmol})$, and MOM-MAC (52.5 mg, $0.42 \mathrm{mmol})$ for $18 \mathrm{~h}$. Purification by flash column chromatography $\left(\mathrm{SiO}_{2}, 10 \rightarrow 15 \%\right.$ EtOAc/Hexanes) afforded $\mathbf{3 h}(56.0 \mathrm{mg}, 82 \%)$ as a white crystalline solid.

Analytical data for $\mathbf{3 h}$ :

${ }^{1} \mathbf{H}$ NMR $\left(500 \mathrm{MHz}, \mathrm{CDCl}_{3}\right): \delta 5.08(\mathrm{~s}, 2 \mathrm{H}), 3.56(\mathrm{~s}, 3 \mathrm{H}), 3.14(\mathrm{~d}, J=2.6 \mathrm{~Hz}, 2 \mathrm{H}), 2.40$ (t, $J=2.7 \mathrm{~Hz}, 1 \mathrm{H})$

${ }^{13}$ C NMR (125 MHz, $\left.\mathrm{CDCl}_{3}\right){ }^{13} \mathrm{C}$ NMR $\left(125 \mathrm{MHz}, \mathrm{CDCl}_{3}\right): \delta 112.2(\mathrm{x} 2), 96.5,75.3$, $73.2,64.9,57.4,31.5$

IR (ATR): 1164, 1037, 978, 922, 666, 616

HRMS (EI) calcd for $\left(\mathrm{C}_{8} \mathrm{H}_{7} \mathrm{O}_{2} \mathrm{~N}_{2}\right)^{+}[\mathrm{M}-\mathrm{H}]^{+}:$163.0508. Found: 163.0512

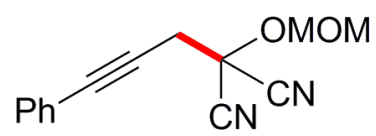

$3 \mathbf{i}$

2-(Methoxymethoxy)-2-(3-phenylprop-2-yn-1-yl)malononitrile $\quad$ (3i): $\quad$ Prepared according to the general procedure using $\mathrm{PPh}_{3}(157 \mathrm{mg}, 0.60 \mathrm{mmol})$, THF $(2.5 \mathrm{~mL})$, DIAD (118 $\mu \mathrm{L}, 0.60 \mathrm{mmol}), 3$-phenyl-2-propyn-1-ol (75 $\mu \mathrm{L}, 0.60 \mathrm{mmol})$, and MOMMAC ( $50.4 \mathrm{mg}, 0.40 \mathrm{mmol})$ for $3 \mathrm{~h}$. Purification by flash column chromatography $\left(\mathrm{SiO}_{2}\right.$, $14 \%$ EtOAc/Hexanes) afforded 3i (87.2 mg, 91\%) as an amber oil.

Analytical data for 3i:

${ }^{1} \mathbf{H}$ NMR $\left(500 \mathrm{MHz}, \mathrm{CDCl}_{3}\right): \delta 7.48(\mathrm{dd}, J=8.1,1.7 \mathrm{~Hz}, 2 \mathrm{H}), 7.39-7.30(\mathrm{~m}, 3 \mathrm{H}), 5.10$ $(\mathrm{s}, 2 \mathrm{H}), 3.57(\mathrm{~s}, 3 \mathrm{H}), 3.36(\mathrm{~s}, 2 \mathrm{H})$

${ }^{13}$ C NMR (125 MHz, $\left.\mathrm{CDCl}_{3}\right): \delta 131.9$ (x2), 128.9, 128.3 (x2), 121.7, 112.5 (x2), 96.4, $86.9,78.3,65.3,57.4,32.4$

IR (ATR): 1491, 1443, 1216, 1164, 1084, 1035, 968, 919, 756, 690

HRMS (FAB) calcd for $\left(\mathrm{C}_{14} \mathrm{H}_{12} \mathrm{O}_{2} \mathrm{~N}_{2} \mathrm{Na}\right)^{+}[\mathrm{M}+\mathrm{Na}]^{+}: 263.0796$. Found: 263.0789 


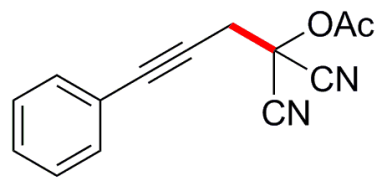

$3 \mathbf{i}^{\prime}$

1,1-Dicyano-4-phenylbut-3-yn-1-yl acetate (3i'): Prepared according to the general procedure using $\mathrm{PPh}_{3}(115 \mathrm{mg}, 0.44 \mathrm{mmol})$, THF $(1 \mathrm{~mL})$, DIAD $(87 \mu \mathrm{L}, 0.44 \mathrm{mmol}), 3-$ phenyl-2-propyn-1-ol ( $55 \mu \mathrm{L}, 0.44 \mathrm{mmol})$, and MOM-MAC (49 mg, $0.4 \mathrm{mmol}$ ) for $48 \mathrm{~h}$. Purification by flash column chromatography $\left(\mathrm{SiO}_{2}, 25 \%\right.$ EtOAc/Hexanes) and recycling preparative HPLC afforded 3i' (64 mg, 68\%) as a colorless oil.

Analytical data for 3i':

${ }^{1} \mathbf{H}$ NMR $\left(600 \mathrm{MHz} ; \mathrm{CDCl}_{3}\right): \delta 7.50-7.47(\mathrm{~m}, 2 \mathrm{H}), 7.39-7.31(\mathrm{~m}, 3 \mathrm{H}), 3.46(\mathrm{~s}, 2 \mathrm{H})$, $2.27(\mathrm{~s}, 3 \mathrm{H})$

${ }^{13}$ C NMR (150 MHz; $\left.\mathrm{CDCl}_{3}\right): \delta 167.3,131.9$ (x2), 129.2, 128.4 (x2), 121.4, 111.2 (x2), $87.5(\mathrm{x} 2), 60.9,30.9,20.0$

IR (ATR): 1781, 1491, 1372, 1199, 1050, 758, 691

HRMS (FAB) calcd for $\left(\mathrm{C}_{14} \mathrm{H}_{11} \mathrm{O}_{2} \mathrm{~N}_{2}\right)[\mathrm{M}+\mathrm{H}]^{+}: 239.0820$. Found: 239.0828

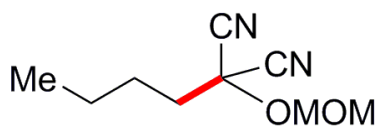

3j

2-Butyl-2-(methoxymethoxy)malononitrile (3j): Prepared according to modified general procedure using $\mathrm{PPh}_{3}(115 \mathrm{mg}, 0.44 \mathrm{mmol})$, THF $(1 \mathrm{~mL})$, DIAD $(87 \mu \mathrm{L}, 0.44$ mmol), 1-butanol ( $40 \mu \mathrm{L}, 0.44 \mathrm{mmol}$ ), and MOM-MAC (50 mg, $0.4 \mathrm{mmol})$ for $7 \mathrm{~h}$. Purification by flash column chromatography $\left(\mathrm{SiO}_{2}, 15 \%\right.$ EtOAc/Hexanes) afforded $\mathbf{3 j}$ (65 $\mathrm{mg}, 89 \%$ ) as a pale yellow (almost colorless) oil.

Analytical data for $\mathbf{3 j}$ :

${ }^{1} \mathbf{H}$ NMR $\left(600 \mathrm{MHz}, \mathrm{CDCl}_{3}\right): \delta 5.04(\mathrm{~s}, 2 \mathrm{H}), 3.54(\mathrm{~s}, 3 \mathrm{H}), 2.22-2.19(\mathrm{~m}, 2 \mathrm{H}), 1.68-$ $1.62(\mathrm{~m}, 2 \mathrm{H}), 1.45(\mathrm{sextet}, J=7.2 \mathrm{~Hz}, 2 \mathrm{H}), 0.98(\mathrm{t}, J=7.2 \mathrm{~Hz}, 3 \mathrm{H})$

${ }^{13}$ C NMR (150 MHz, $\left.\mathrm{CDCl}_{3}\right): \delta 113.3$ (x2), 96.1, 65.8, 57.3, 39.8, 25.6, 21.8, 13.6

IR (ATR): 1458, 1166, 1091, 1008, 965, 923

HRMS (EI) calcd for $\left(\mathrm{C}_{9} \mathrm{H}_{13} \mathrm{O}_{2} \mathrm{~N}_{2}\right)^{+}[\mathrm{M}-\mathrm{H}]^{+}:$181.0977. Found: 181.0974 


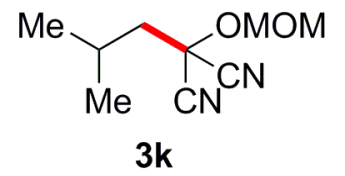

2-Isobutyl-2-(methoxymethoxy)malononitrile (3k): Prepared according to the general procedure using $\mathrm{PPh}_{3}(115 \mathrm{mg}, 0.44 \mathrm{mmol})$, THF $(2 \mathrm{~mL})$, DIAD (87 $\left.\mu \mathrm{L}, 0.44 \mathrm{mmol}\right)$, isobutyl alcohol $(41 \mu \mathrm{L}, 0.44 \mathrm{mmol})$, and MOM-MAC (50 mg, $0.4 \mathrm{mmol})$ for $2 \mathrm{~h}$. Purification by flash column chromatography $\left(\mathrm{SiO}_{2}, 14 \%\right.$ EtOAc/Hexanes) afforded 3k (50 $\mathrm{mg}, 69 \%)$ as a colorless oil.

Analytical data for $\mathbf{3 k}$ :

${ }^{1} \mathbf{H}$ NMR $\left(500 \mathrm{MHz}, \mathrm{CDCl}_{3}\right): \delta 5.05$ (s, 2H), $3.54(\mathrm{~s}, 3 \mathrm{H}), 2.18-2.07$ (m, 3H), 1.10 (d, J $=6.6 \mathrm{~Hz}, 6 \mathrm{H})$

${ }^{13}$ C NMR (125 MHz, $\left.\mathrm{CDCl}_{3}\right): \delta 113.5(\mathrm{x} 2), 96.1,65.2,57.3,47.8,25.1,23.1(\mathrm{x} 2)$

IR (ATR): 2964, 1470, 1165, 1088, 1005, 966, 923

HRMS (EI) calcd for $\left(\mathrm{C}_{9} \mathrm{H}_{13} \mathrm{O}_{2} \mathrm{~N}_{2}\right)[\mathrm{M}-\mathrm{H}]^{+}:$181.0977. Found: 181.0981

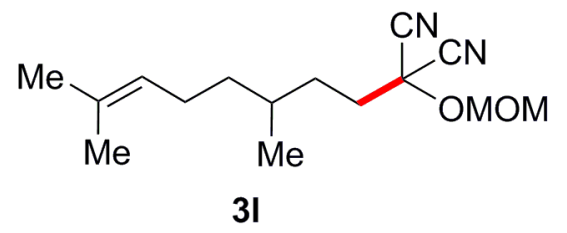

2-(3,7-Dimethyloct-6-en-1-yl)-2-(methoxymethoxy)malononitrile $\quad$ (3I): $\quad$ Prepared according to the general procedure using $\mathrm{PPh}_{3}(115 \mathrm{mg}, 0.44 \mathrm{mmol})$, THF (1 mL), DIAD (87 $\mu \mathrm{L}, 0.44 \mathrm{mmol}), \beta$-citronellol $(80 \mu \mathrm{L}, 0.44 \mathrm{mmol})$, and MOM-MAC (50 mg, 0.4 $\mathrm{mmol})$ for $2 \mathrm{~h}$. Purification by flash column chromatography $\left(\mathrm{SiO}_{2}, 9 \%\right.$ EtOAc/Hexanes) afforded 31 (81 $\mathrm{mg}, 77 \%)$ as a colorless oil.

Analytical data for 31:

${ }^{1} \mathbf{H}$ NMR $\left(600 \mathrm{MHz}, \mathrm{CDCl}_{3}\right): \delta 5.08(\mathrm{tt}, J=6.6,1.2 \mathrm{~Hz}, 1 \mathrm{H}), 5.04(\mathrm{~s}, 2 \mathrm{H}), 3.54(\mathrm{~s}, 3 \mathrm{H})$, $2.24(\mathrm{td}, J=13,4.8 \mathrm{~Hz}, 1 \mathrm{H}), 2.17$ (ddd, $J=14,12,4.8 \mathrm{~Hz}, 1 \mathrm{H}), 2.03$ (sextet, $J=7.2 \mathrm{~Hz}$, 1H), 1.97 (sextet, $J=7.2 \mathrm{~Hz}, 1 \mathrm{H}), 1.69(\mathrm{~s}, 3 \mathrm{H}), 1.69-1.65(\mathrm{~m}, 1 \mathrm{H}), 1.61(\mathrm{~s}, 3 \mathrm{H}), 1.57-$ $1.46(\mathrm{~m}, 2 \mathrm{H}), 1.39-1.34(\mathrm{~m}, 1 \mathrm{H}), 1.25-1.19(\mathrm{~m}, 1 \mathrm{H}), 0.94(\mathrm{~d}, J=6 \mathrm{~Hz}, 3 \mathrm{H})$

${ }^{13}$ C NMR (150 MHz, $\left.\mathrm{CDCl}_{3}\right): \delta 131.7,124.2,113.3(\mathrm{x} 2), 96.2,66.0,57.3,38.0,36.5$, $31.7,30.3,25.7,25.3,19.2,17.6$

IR (ATR): 2920, 1455, 1165, 1088, 1028, 924

HRMS (FAB) calcd for $\left(\mathrm{C}_{15} \mathrm{H}_{23} \mathrm{O}_{2} \mathrm{~N}_{2}\right)[\mathrm{M}-\mathrm{H}]^{+}:$263.1759. Found: 263.1756 


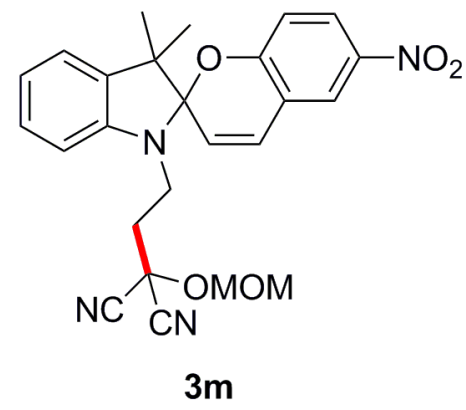

2-(2-(3',3'-Dimethyl-6-nitrospiro[chromene-2,2'-indolin]-1'-yl)ethyl)-2-

(methoxymethoxy)malononitrile (3m): Prepared according to the general procedure using $\mathrm{PPh}_{3}$ (164 mg, $\left.0.63 \mathrm{mmol}\right)$, THF (2.3 mL), DIAD (123 $\left.\mu \mathrm{L}, 0.63 \mathrm{mmol}\right), 2-\left(3^{\prime}, 3^{\prime}-\right.$ dimethyl-6-nitrospiro[chromene-2,2'-indolin]-1'-yl)ethan-1-ol (221 mg, $0.63 \mathrm{mmol})$, and MOM-MAC (53 mg, $0.42 \mathrm{mmol}$ ) for $6 \mathrm{~h}$. Purification by flash column chromatography $\left(\mathrm{SiO}_{2}, 20 \% \mathrm{EtOAc} / \mathrm{Hexanes}\right)$ afforded 3m (161 mg, 84\%) as a purple oil.

Analytical data for $\mathbf{3 m}$ :

${ }^{1} \mathbf{H}$ NMR $\left(600 \mathrm{MHz} ; \mathrm{CDCl}_{3}\right): \delta 8.05(\mathrm{dd}, J=8.4,3.0 \mathrm{~Hz}, 1 \mathrm{H}), 8.03(\mathrm{~d}, J=3.0 \mathrm{~Hz}, 1 \mathrm{H})$, $7.23(\mathrm{t}, J=7.2 \mathrm{~Hz}, 1 \mathrm{H}), 7.13(\mathrm{~d}, J=3.6 \mathrm{~Hz}, 1 \mathrm{H}), 6.98(\mathrm{~d}, J=6.0 \mathrm{~Hz}, 1 \mathrm{H}), 6.94(\mathrm{t}, J=7.2$ $\mathrm{Hz}, 1 \mathrm{H}), 6.77(\mathrm{~d}, J=9.0 \mathrm{~Hz}, 1 \mathrm{H}), 6.64(\mathrm{~d}, J=8.4 \mathrm{~Hz}, 1 \mathrm{H}), 5.89(\mathrm{~d}, J=11 \mathrm{~Hz}, 1 \mathrm{H}), 5.02$ (s, 2H), $3.66(\mathrm{ddd}, J=15,10,6.0 \mathrm{~Hz}, 1 \mathrm{H}), 3.54(\mathrm{ddd}, J=14,10,5.4 \mathrm{~Hz}, 1 \mathrm{H}), 3.51$ (s, $3 \mathrm{H}), 2.62(\mathrm{ddd}, J=12,9.6,4.8 \mathrm{~Hz}, 1 \mathrm{H}), 2.46(\mathrm{ddd}, J=15,10,6.0 \mathrm{~Hz}, 1 \mathrm{H}), 1.29(\mathrm{~s}, 3 \mathrm{H})$, $1.19(\mathrm{~s}, 3 \mathrm{H})$

${ }^{13}$ C NMR (150 MHz; $\left.\mathrm{CDCl}_{3}\right): \delta 159.0,145.7,141.3,136.0,128.9,127.9,126.1,122.9$, $122.1,121.4,120.5,118.4,115.5,112.79,112.76,106.6,106.5,96.3,63.6,57.5,52.9$, $38.3,38.1,25.7,19.9$

IR (ATR):2966, 1609, 1577, 1518, 1479, 1458, 1335, 1268, 1165, 1087, 993, 955, 919, $809,743,680$

HRMS (EI) calcd for $\left(\mathrm{C}_{25} \mathrm{H}_{25} \mathrm{O}_{5} \mathrm{~N}_{4}\right)[\mathrm{M}+\mathrm{H}]^{+}: 461.1825$. Found: 461.1844

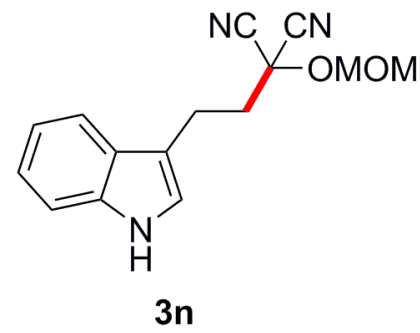

2-(2-(1H-Indol-3-yl)ethyl)-2-(methoxymethoxy)malononitrile $\quad(3 n): \quad$ Prepared according to the general procedure using $\mathrm{PPh}_{3}(131 \mathrm{mg}, 0.50 \mathrm{mmol})$, THF $(1.5 \mathrm{~mL})$, DIAD (98 $\mu \mathrm{L}, 0.50 \mathrm{mmol})$, tryptophol $(80 \mathrm{mg}, 0.50 \mathrm{mmol})$, and MOM-MAC (57 $\mathrm{mg}$, $0.45 \mathrm{mmol})$ for $20 \mathrm{~h}$. Purification by flash column chromatography $\left(\mathrm{SiO}_{2}, 20 \%\right.$ EtOAc/Hexanes) afforded 3n (81 mg, 66\%) as a white solid. 
Analytical data for $\mathbf{3 n}$ :

${ }^{1} \mathbf{H}$ NMR $\left(600 \mathrm{MHz}, \mathrm{CDCl}_{3}\right): \delta 8.03($ br s, $1 \mathrm{H}), 7.60(\mathrm{~d}, J=7.2 \mathrm{~Hz}, 1 \mathrm{H}), 7.37(\mathrm{~d}, J=8.4$ $\mathrm{Hz}, 1 \mathrm{H}), 7.23(\mathrm{t}, J=7.2 \mathrm{~Hz}, 1 \mathrm{H}), 7.16(\mathrm{t}, J=7.2 \mathrm{~Hz}, 1 \mathrm{H}), 7.04(\mathrm{~d}, J=2.4 \mathrm{~Hz}, 1 \mathrm{H}), 5.09$ $(\mathrm{s}, 2 \mathrm{H}), 3.57(\mathrm{~s}, 3 \mathrm{H}), 3.16-3.13(\mathrm{~m}, 2 \mathrm{H}), 2.60-2.57(\mathrm{~m}, 2 \mathrm{H})$

${ }^{13} \mathrm{C}$ NMR (150 MHz, $\left.\mathrm{CDCl}_{3}\right): \delta 136.3,126.8,122.4,121.8,119.7,118.3,113.2(\mathrm{x} 2)$, $112.5,111.3,96.2,65.4,57.4,40.6,19.6$

IR (ATR): 3418, 1456, 1337, 1219, 1163, 1091, 1035, 984, 921, 740, 633, 619, 604

HRMS (ESI) calcd for $\left(\mathrm{C}_{15} \mathrm{H}_{16} \mathrm{O}_{2} \mathrm{~N}_{3}\right)[\mathrm{M}+\mathrm{H}]^{+}:$270.1237. Found: 270.1231

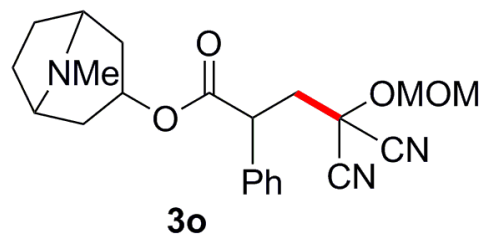

8-Methyl-8-azabicyclo[3.2.1]octan-3-yl 4,4-dicyano-4-(methoxymethoxy)-2phenylbutanoate (3o): Prepared according to the general procedure using $\mathrm{PPh}_{3}(177 \mathrm{mg}$, $0.68 \mathrm{mmol})$, THF (2.5 mL), DIAD (133 $\mu \mathrm{L}, 0.68 \mathrm{mmol})$, atropine (195 mg, $0.68 \mathrm{mmol})$, and MOM-MAC (57 mg, $0.45 \mathrm{mmol})$ for $16 \mathrm{~h}$. Purification by flash column chromatography $\left(\mathrm{SiO}_{2}, 5 \rightarrow 20 \% \mathrm{MeOH} / \mathrm{CH}_{2} \mathrm{Cl}_{2}\right)$ afforded 3o (155 $\left.\mathrm{mg}, 86 \%\right)$ as a colorless oil.

Analytical data for 3o:

${ }^{1} \mathbf{H}$ NMR $\left(600 \mathrm{MHz} ; \mathrm{CDCl}_{3}\right): \delta 7.38-7.30(\mathrm{~m}, 5 \mathrm{H}), 5.02-4.98(\mathrm{~m}, 3 \mathrm{H}), 3.90(\mathrm{dd}, J=$ 8.4, $5.4 \mathrm{~Hz}, 1 \mathrm{H}), 3.51$ (s, 3H), 3.30, (dd, $J=14,8.4 \mathrm{~Hz}, 1 \mathrm{H}), 3.05$ (t, $J=3.6 \mathrm{~Hz}, 1 \mathrm{H})$, $2.92(\mathrm{t}, J=3.0 \mathrm{~Hz}, 1 \mathrm{H}), 2.62(\mathrm{dd}, J=14,5.4 \mathrm{~Hz}, 1 \mathrm{H}), 2.21$ (s $3 \mathrm{H}), 2.11$ (dt, $J=16,4.2$ $\mathrm{Hz}, 1 \mathrm{H}), 2.01(\mathrm{dt}, J=14,4.2 \mathrm{~Hz}, 1 \mathrm{H}), 1.94-1.88(\mathrm{~m}, 1 \mathrm{H}), 1.79-1.71(\mathrm{~m}, 2 \mathrm{H}), 1.69(\mathrm{~d}$, $J=16 \mathrm{~Hz}, 1 \mathrm{H}), 1.43(\mathrm{~d}, J=14 \mathrm{~Hz}, 1 \mathrm{H}), 1.24-1.20(\mathrm{~m}, 1 \mathrm{H})$

${ }^{13}$ C NMR (150 MHz; $\left.\mathrm{CDCl}_{3}\right): \delta 170.7,136.6,129.2$ (x2), 128.4, 127.8 (x2), 112.61, $112.57,96.2,69.1,64.3,59.6,59.5,57.5,47.1,42.2,40.4,36.4,36.2,25.4,25.0$

IR (ATR): 2938, 1727, 1201, 1167, 1033, 983, 698

HRMS (ESI) calcd for $\left(\mathrm{C}_{22} \mathrm{H}_{28} \mathrm{O}_{4} \mathrm{~N}_{3}\right)[\mathrm{M}+\mathrm{H}]^{+}:$398.2080. Found: 398.2068

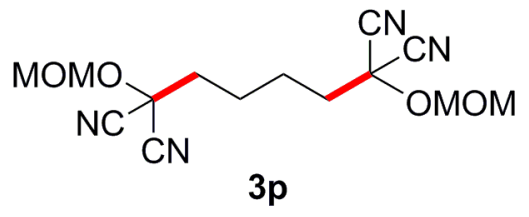

2,4,11,13-Tetraoxatetradecane-5,5,10,10-tetracarbonitrile (3p): Prepared according to the general procedure using $\mathrm{PPh}_{3}(115 \mathrm{mg}, 0.44 \mathrm{mmol})$, THF (2 mL), DIAD (87 $\mu \mathrm{L}, 0.44$ 
mmol), 1,4-butanediol ( $20 \mu \mathrm{L}, 0.22 \mathrm{mmol}$ ), and MOM-MAC (48.1 $\mathrm{mg}, 0.38 \mathrm{mmol})$ for 3 h. Purification by flash column chromatography $\left(\mathrm{SiO}_{2}, \mathrm{CH}_{2} \mathrm{Cl}_{2}\right)$ afforded $\mathbf{3 p}(28.1 \mathrm{mg}$, $48 \%)$ as a colorless solid.

Analytical data for 3p:

${ }^{1} \mathbf{H}$ NMR $\left(500 \mathrm{MHz}, \mathrm{CDCl}_{3}\right): \delta 5.05(\mathrm{~s}, 4 \mathrm{H}), 3.55(\mathrm{~s}, 6 \mathrm{H}), 2.35-2.21(\mathrm{~m}, 4 \mathrm{H}), 1.81$ (quintet, $J=3.7 \mathrm{~Hz}, 4 \mathrm{H}$ )

${ }^{13} \mathrm{C}$ NMR (125 MHz, $\mathrm{CDCl}_{3}$ ): $\delta 113.0$ (x4), 96.3 (x2), 65.3 (x2), 57.4 (x2), 39.6 (x2), $22.8(\mathrm{x} 2)$

IR (ATR): 1469, 1254, 1227, 1199, 1166, 1091, 997, 962, 922, 858, 700

HRMS (FAB) calcd for $\left(\mathrm{C}_{14} \mathrm{H}_{18} \mathrm{O}_{4} \mathrm{~N}_{4}\right) \mathrm{H}^{+}[\mathrm{M}+\mathrm{H}]^{+}:$307.1401. Found: 307.1396

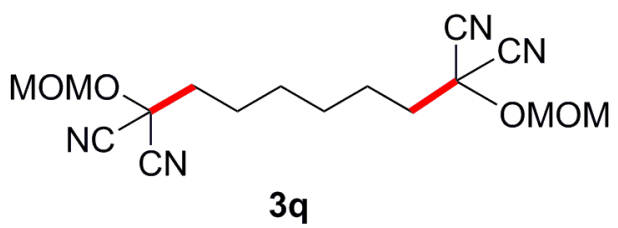

2,4,13,15-Tetraoxahexadecane-5,5,12,12-tetracarbonitrile (3q): Prepared according to modified general procedure using $\mathrm{PPh}_{3}(115 \mathrm{mg}, 0.44 \mathrm{mmol})$, THF $(1 \mathrm{~mL})$, DIAD (87 $\mu \mathrm{L}, 0.44 \mathrm{mmol}$ ), 1,6-hexanediol (28 mg, $0.22 \mathrm{mmol}$ ), and MOM-MAC (50 mg, 0.4 mmol) for $8 \mathrm{~h}$. Purification by flash column chromatography $\left(\mathrm{SiO}_{2}, 15 \%\right.$ EtOAc/Hexanes) afforded $\mathbf{3 q}$ (41 $\mathrm{mg}, 61 \%)$ as a white solid and 3q' (20 $\mathrm{mg}, 40 \%)$ as a pale yellow oil.

Analytical data for 3q:

${ }^{1} \mathbf{H}$ NMR $\left(500 \mathrm{MHz}, \mathrm{CDCl}_{3}\right): \delta 5.04(\mathrm{~s}, 4 \mathrm{H}), 3.54(\mathrm{~s}, 6 \mathrm{H}), 2.27-2.16(\mathrm{~m}, 4 \mathrm{H}), 1.73-$ 1.67(m, 4H), 1.49 (quintet, $J=3.5 \mathrm{~Hz}, 4 \mathrm{H}$ )

${ }^{13} \mathrm{C}$ NMR (125 MHz, $\left.\mathrm{CDCl}_{3}\right): \delta 113.2$ (x4), 96.2 (x2), $65.6(\mathrm{x} 2), 57.3(\mathrm{x} 2), 39.9$ (x2), $28.2(\mathrm{x} 2), 23.4(\mathrm{x} 2)$

IR (ATR): 2943, 1219, 1159, 1084, 1019, 988, 917, 666, 616

HRMS (FAB) calcd for $\left(\mathrm{C}_{16} \mathrm{H}_{22} \mathrm{O}_{4} \mathrm{~N}_{4} \mathrm{Na}\right)^{+}[\mathrm{M}+\mathrm{Na}]^{+}: 357.1539$. Found: 357.1529

Analytical data for $\mathbf{3 q}$ :

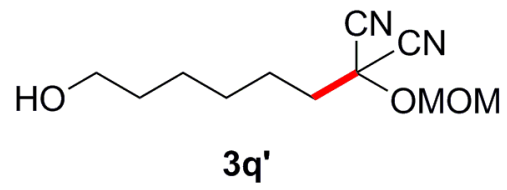

${ }^{1} \mathbf{H}$ NMR $\left(500 \mathrm{MHz}, \mathrm{CDCl}_{3}\right): \delta 5.03(\mathrm{~s}, 2 \mathrm{H}), 3.66(\mathrm{t}, J=6.5 \mathrm{~Hz}, 2 \mathrm{H}), 3.54(\mathrm{~s}, 3 \mathrm{H}), 2.25-$ $2.17(\mathrm{~m}, 2 \mathrm{H}), 1.74-1.64(\mathrm{~m}, 2 \mathrm{H}), 1.60$ (quintet, $J=6.7 \mathrm{~Hz}, 2 \mathrm{H}), 1.50-1.41(\mathrm{~m}, 4 \mathrm{H})$ 
${ }^{13}$ C NMR (125 MHz, $\left.\mathrm{CDCl}_{3}\right): \delta 113.3$ (x2), 96.1, 65.8, 62.6, 57.3, 40.0, 32.3, 28.4, 25.3, 23.6

GCMS calcd for $\left(\mathrm{C}_{16} \mathrm{H}_{22} \mathrm{O}_{4} \mathrm{~N}_{4}\right)^{*}$ [M-MAC] : 209. Found: 209

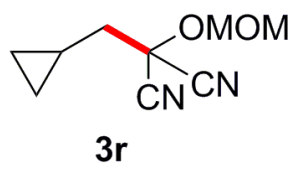

2-(Cyclopropylmethyl)-2-(methoxymethoxy)malononitrile (3r): Prepared according to the general procedure using $\mathrm{PPh}_{3}(107 \mathrm{mg}, 0.41 \mathrm{mmol})$, THF $(2 \mathrm{~mL})$, DIAD ( $80 \mu \mathrm{L}, 0.41$ mmol), cyclopropylmethanol (33 $\mathrm{mg}, 0.41 \mathrm{mmol}$ ), and MOM-MAC (42.9 $\mathrm{mg}, 0.34$ mmol) for $4 \mathrm{~h}$. Purification by flash column chromatography $\left(\mathrm{SiO}_{2}, 10 \%\right.$ EtOAc/Hexanes) afforded $\mathbf{3 r}(49 \mathrm{mg}, 80 \%)$ as a colorless oil.

Analytical data for 3r:

${ }^{1} \mathbf{H}$ NMR (600 MHz; $\left.\mathrm{CDCl}_{3}\right): \delta 5.06(\mathrm{~s}, 2 \mathrm{H}), 3.55(\mathrm{~s}, 3 \mathrm{H}), 2.15(\mathrm{~d}, J=7.2 \mathrm{~Hz}, 2 \mathrm{H}), 1.09$ $1.02(\mathrm{~m}, 2 \mathrm{H}), 0.75-0.66(\mathrm{~m}, 2 \mathrm{H}), 0.45-0.37(\mathrm{~m}, 2 \mathrm{H})$

${ }^{13}$ C NMR (150 MHz; $\left.\mathrm{CDCl}_{3}\right): \delta 113.4(\mathrm{x} 2), 96.0,65.8,57.2,44.6,5.3,4.3(\mathrm{x} 2)$

IR (ATR):1217, 1162, 1086, 1024, 981, 922, 863, 831, 800, 694

HRMS (EI) calcd for $\left(\mathrm{C}_{9} \mathrm{H}_{11} \mathrm{O}_{2} \mathrm{~N}_{2}\right)[\mathrm{M}-\mathrm{H}]^{+}:$179.0821. Found: 179.0828

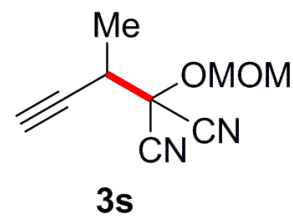

2-(But-3-yn-2-yl)-2-(methoxymethoxy)malononitrile (3s): Prepared according to the general procedure using $\mathrm{PPh}_{3}(115 \mathrm{mg}, 0.44 \mathrm{mmol})$, THF $(1 \mathrm{~mL})$, DIAD $(87 \mu \mathrm{L}, 0.44$ mmol), 3-butyn-2-ol (34 $\mu \mathrm{L}, 0.44 \mathrm{mmol})$, and MOM-MAC (50 mg, $0.4 \mathrm{mmol})$ for $8 \mathrm{~h}$. Purification by flash column chromatography $\left(\mathrm{SiO}_{2}, 10 \%\right.$ EtOAc/Hexanes) afforded 3s (48 $\mathrm{mg}, 68 \%)$ as a colorless oil.

Analytical data for 3s:

${ }^{1} \mathbf{H}$ NMR $\left(600 \mathrm{MHz}, \mathrm{CDCl}_{3}\right): \delta 5.09(\mathrm{~d}, J=7.2 \mathrm{~Hz}, 1 \mathrm{H}), 5.08(\mathrm{~d}, J=7.2 \mathrm{~Hz}, 1 \mathrm{H}), 3.56(\mathrm{~s}$, $3 \mathrm{H}), 3.25(\mathrm{qd}, J=7.2,2.4 \mathrm{~Hz}, 1 \mathrm{H}), 2.45(\mathrm{~d}, J=2.4 \mathrm{~Hz}, 1 \mathrm{H}), 1.53(\mathrm{~d}, J=7.2 \mathrm{~Hz}, 3 \mathrm{H})$

${ }^{13}$ C NMR $\left(150 \mathrm{MHz}, \mathrm{CDCl}_{3}\right): \delta 112.2,111.8,96.6,78.7,74.8,69.4,57.5,37.1,15.6$

IR (ATR): 3295, 1456, 1218, 1165, 1108, 1041, 932, 657 
HRMS (EI) calcd for $\left(\mathrm{C}_{9} \mathrm{H}_{9} \mathrm{O}_{2} \mathrm{~N}_{2}\right)^{+}[\mathrm{M}-\mathrm{H}]^{+}$: 177.0664. Found: 177.0672

\section{General Procedure for Unmasking of the Mitsunobu Adducts}

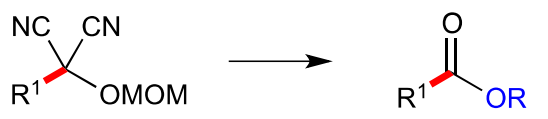

To an oven-dried two-neck round bottom flask was added MAC adduct 3 (1 equiv), 1:1 $\mathrm{AcOH} / \mathrm{DME}(0.5 \mathrm{M})$, and $(R)$-CSA (0.5-1.1 equiv). The flask was sealed with two glass stoppers and heated to $60{ }^{\circ} \mathrm{C}$ for $3-8 \mathrm{~h}$ until the conversion to cyanohydrin was complete as determined by TLC. At this point one of the two stoppers was changed to a septum and the other was connected to nitrogen atmosphere. The reaction mixture was cooled to ambient temperature, diluted with anhydrous $\mathrm{MeOH}(0.5 \mathrm{M})$, and cooled to $-40{ }^{\circ} \mathrm{C}$. A solution of 1:1 $\mathrm{MeOH} / \mathrm{Et}_{3} \mathrm{~N}$ (20.4 equiv. of $\mathrm{Et}_{3} \mathrm{~N}$ ) was added dropwise over $10 \mathrm{~min}$. After 2-3 $\mathrm{h}$, the reaction was warmed to $0{ }^{\circ} \mathrm{C}$. After $3-15 \mathrm{~h}$ at $0{ }^{\circ} \mathrm{C}$, the reaction was slowly quenched with sat. aq. $\mathrm{NH}_{4} \mathrm{Cl}$. The mixture was extracted three times with $\mathrm{CH}_{2} \mathrm{Cl}_{2}$ and the combined organics were washed with brine, dried over anh. $\mathrm{Na}_{2} \mathrm{SO}_{4}$, and concentrated. Purification by flash column chromatography $\left(\mathrm{SiO}_{2}, \mathrm{EtOAc} / \mathrm{Hexanes}\right)$ afforded ester $\mathbf{5}$.

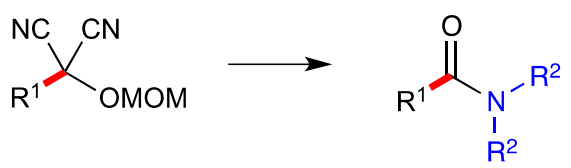

To an oven-dried two-neck round bottom flask was added MAC adduct 3 (1 equiv), 1:1 $\mathrm{AcOH} / \mathrm{DME}(0.5 \mathrm{M})$, and $(R)$-CSA (1.1 equiv). The flask was sealed with two glass stoppers and heated to $60^{\circ} \mathrm{C}$ for $5.5 \mathrm{~h}$ until the conversion to cyanohydrin was complete as determined by TLC. At this point one of the two stoppers was changed to a septum and the other was connected to nitrogen atmosphere. The reaction mixture was cooled to ambient temperature, diluted with $\mathrm{CH}_{2} \mathrm{Cl}_{2}(0.5 \mathrm{M})$, and cooled to $-40{ }^{\circ} \mathrm{C}$. A solution of 1:1 Amine/ $\mathrm{CH}_{2} \mathrm{Cl}_{2}$ (20.4 equiv of amine) was added dropwise over 10 min, then $\mathrm{Et}_{3} \mathrm{~N}$ (20.4 equiv) was added. After $10 \mathrm{~min}$, the reaction was warmed to $0{ }^{\circ} \mathrm{C}$. After $0.4-2 \mathrm{~h}$ at 0 ${ }^{\circ} \mathrm{C}$, the reaction was slowly quenched with sat. aq. $\mathrm{NH}_{4} \mathrm{Cl}$. The mixture was extracted three times with $\mathrm{CH}_{2} \mathrm{Cl}_{2}$ and the combined organics were dried over anh. $\mathrm{Na}_{2} \mathrm{SO}_{4}$ and concentrated. Purification by flash column chromatography $\left(\mathrm{SiO}_{2}\right.$, EtOAc/Hexanes $\rightarrow \mathrm{MeOH} / \mathrm{CHCl}_{3}$ ) afforded amide $\mathbf{6}$.<smiles>[R]OC([R])(C#N)OOC</smiles>

To an oven-dried 2-dram vial was added MAC adduct 3 (1 equiv), $(R)$-CSA (0.5-5 equiv) and $1: 1 \mathrm{AcOH} / \mathrm{H}_{2} \mathrm{O}(0.2 \mathrm{M})$. The vial was sealed with a cap and heated to $60{ }^{\circ} \mathrm{C}$ for $15-$ 
$19 \mathrm{~h}$. The reaction mixture was cooled to ambient temperature. The mixture was extracted three times with $\mathrm{CH}_{2} \mathrm{Cl}_{2}$ and the combined organics were dried over anh. $\mathrm{Na}_{2} \mathrm{SO}_{4}$ and concentrated. Purification by flash column chromatography $\left(\mathrm{SiO}_{2}\right.$, $\mathrm{MeOH} / \mathrm{CHCl}_{3}$ ) afforded carboxylic acid 7.

Unmasked Esters, Amides, and Carboxylic Acids<smiles>COC(=O)CCc1ccccc1</smiles>

Methyl 3-phenylpropanoate (5a): Prepared according to the general procedure using MAC adduct 3a $(61 \mathrm{mg}, 0.26 \mathrm{mmol}), 1: 1 \mathrm{AcOH} / \mathrm{DME}(0.53 \mathrm{~mL})$, and $(R)-\mathrm{CSA}(30 \mathrm{mg}$, $0.13 \mathrm{mmol})$ for $3 \mathrm{~h}$, then anhydrous $\mathrm{MeOH}(0.53 \mathrm{~mL})$ and 1:1 $\mathrm{MeOH} / \mathrm{Et}_{3} \mathrm{~N}(1.5 \mathrm{~mL}, 5.4$ mmol of $\left.\mathrm{Et}_{3} \mathrm{~N}\right)$. Purification by flash column chromatography $\left(\mathrm{SiO}_{2}, 10 \%\right.$ EtOAc/Hexanes) afforded $\mathbf{5 a}(35 \mathrm{mg}, 80 \%)$ as a colorless oil.

Analytical data for 5a:

${ }^{1} \mathbf{H}$ NMR $\left(600 \mathrm{MHz} ; \mathrm{CDCl}_{3}\right): \delta 7.29(\mathrm{t}, J=7.8 \mathrm{~Hz}, 2 \mathrm{H}), 7.21-7.19(\mathrm{~m}, 3 \mathrm{H}), 3.67(\mathrm{~s}, 3 \mathrm{H})$, $2.95(\mathrm{t}, J=7.8 \mathrm{~Hz}, 2 \mathrm{H}), 2.63,(\mathrm{t}, J=7.8 \mathrm{~Hz}, 2 \mathrm{H})$

${ }^{13} \mathbf{C ~ N M R}\left(150 \mathrm{MHz} ; \mathrm{CDCl}_{3}\right): \delta 173.3,140.5,128.5,128.2,126.2,51.6,35.7,30.9$

CAS Number: [103-25-3]<smiles>COC(=O)Cc1ccccc1</smiles>

Methyl 2-phenylacetate (5b): Prepared according to the general procedure using MAC adduct $3 \mathrm{~b}(25 \mathrm{mg}, 0.11 \mathrm{mmol}), 1: 1 \mathrm{AcOH} / \mathrm{DME}(0.23 \mathrm{~mL})$, and $(R)$-CSA $(29 \mathrm{mg}, 0.12$ $\mathrm{mmol})$ for $5 \mathrm{~h}$, then anhydrous $\mathrm{MeOH}(0.23 \mathrm{~mL})$ and 1:1 $\mathrm{MeOH} / \mathrm{Et}_{3} \mathrm{~N}(0.65 \mathrm{~mL}, 2.3$ mmol of $\left.\mathrm{Et}_{3} \mathrm{~N}\right)$. Purification by flash column chromatography $\left(\mathrm{SiO}_{2}, 10 \%\right.$ EtOAc/Hexanes) afforded $\mathbf{5 b}(14 \mathrm{mg}, 80 \%)$ as a colorless oil.

Analytical data for $\mathbf{5 b}$ :

${ }^{1} \mathbf{H}$ NMR (600 MHz; $\left.\mathrm{CDCl}_{3}\right): \delta 7.34-7.31(\mathrm{~m}, 2 \mathrm{H}), 7.29-7.25(\mathrm{~m}, 3 \mathrm{H}), 3.69(\mathrm{~s}, 3 \mathrm{H})$, $3.63(\mathrm{~s}, 2 \mathrm{H})$

${ }^{13}$ C NMR (150 MHz; $\left.\mathrm{CDCl}_{3}\right): \delta 172.0,134.0,129.2,128.6,127.1,52.0,41.2$

CAS Number: [101-41-7] 


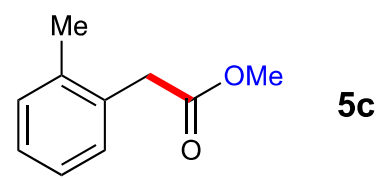

Methyl 2-(2-methyphenyl)acetate (5c): Prepared according to the general procedure using MAC adduct $3 \mathbf{b}(25 \mathrm{mg}, 0.11 \mathrm{mmol}), 1: 1 \mathrm{AcOH} / \mathrm{DME}(0.23 \mathrm{~mL})$, and $(R)$-CSA (29 $\mathrm{mg}, 0.12 \mathrm{mmol})$ for $5 \mathrm{~h}$, then anhydrous $\mathrm{MeOH}(0.23 \mathrm{~mL})$ and $1: 1 \mathrm{MeOH} / \mathrm{Et}_{3} \mathrm{~N}(0.65$ $\mathrm{mL}, 2.3 \mathrm{mmol}$ of $\left.\mathrm{Et}_{3} \mathrm{~N}\right)$. Purification by flash column chromatography $\left(\mathrm{SiO}_{2}, 10 \%\right.$ EtOAc/Hexanes) afforded $\mathbf{5 c}(14 \mathrm{mg}, 80 \%)$ as a colorless oil.

Analytical data for $\mathbf{5 c}$ :

${ }^{1} \mathbf{H}$ NMR (600 MHz; $\left.\mathrm{CDCl}_{3}\right): \delta 7.20-7.15(\mathrm{~m}, 4 \mathrm{H}), 3.69(\mathrm{~s}, 3 \mathrm{H}), 3.64(\mathrm{~s}, 2 \mathrm{H}), 2.31(\mathrm{~s}$, $3 \mathrm{H})$

${ }^{13}$ C NMR $\left(150 \mathrm{MHz} ; \mathrm{CDCl}_{3}\right): \delta 171.9,136.8,132.7,130.3,130.1,127.4,126.1,52.0$, $39.0,19.6$

CAS Number: [7218-49-7]

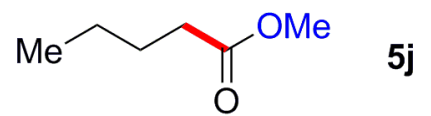

Methyl pentanoate (5j): Prepared according to the general procedure using MAC adduct $3 \mathbf{j}(21 \mathrm{mg}, 0.12 \mathrm{mmol}), 1: 1 \mathrm{AcOH} / \mathrm{DME}(0.23 \mathrm{~mL})$, and $(R)$-CSA (29 mg, $0.13 \mathrm{mmol})$ for $8 \mathrm{~h}$, then anhydrous $\mathrm{MeOH}(0.23 \mathrm{~mL})$ and 1:1 $\mathrm{MeOH} / \mathrm{Et}_{3} \mathrm{~N}\left(0.66 \mathrm{~mL}, 2.4 \mathrm{mmol}^{\circ} \mathrm{Et}_{3} \mathrm{~N}\right)$. Purification by flash column chromatography $\left(\mathrm{SiO}_{2}, 10 \%\right.$ EtOAc/Hexanes) afforded 5j (12 $\mathrm{mg}, 87 \%)$ as a colorless oil.

Analytical data for $\mathbf{5 j}$ :

${ }^{1} \mathbf{H}$ NMR $\left(600 \mathrm{MHz} ; \mathrm{CDCl}_{3}\right): \delta 3.67(\mathrm{~s}, 3 \mathrm{H}), 2.31(\mathrm{t}, J=7.8 \mathrm{~Hz}, 2 \mathrm{H}), 1.61$ (quintet, $J=$ $7.8 \mathrm{~Hz}, 2 \mathrm{H}), 1.35$ (sextet, $J=7.8 \mathrm{~Hz}, 2 \mathrm{H}), 0.92$ (t, $J=7.8 \mathrm{~Hz}, 3 \mathrm{H}$ )

CAS Number: [624-24-8]

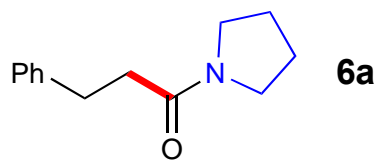

3-Phenyl-1-(pyrrolidin-1-yl)propan-1-one (6a): Prepared according to the general procedure using MAC adduct 3a $(16 \mathrm{mg}, 0.071 \mathrm{mmol}), 1: 1 \mathrm{AcOH} / \mathrm{DME}(0.14 \mathrm{~mL})$, and $(R)$-CSA $(18 \mathrm{mg}, 0.078 \mathrm{mmol})$ for $5.5 \mathrm{~h}$, then anhydrous $\mathrm{CH}_{2} \mathrm{Cl}_{2}(0.14 \mathrm{~mL}), 1: 1$ pyrrolidine $/ \mathrm{CH}_{2} \mathrm{Cl}_{2}(0.24 \mathrm{~mL}, 1.4 \mathrm{mmol}$ of pyrrolidine $)$ and $\mathrm{Et}_{3} \mathrm{~N}(0.20 \mathrm{~mL}, 1.4 \mathrm{mmol})$. Purification by flash column chromatography $\left(\mathrm{SiO}_{2}, 25 \%\right.$ EtOAc/Hexanes $\rightarrow 2 \%$ $\left.\mathrm{MeOH} / \mathrm{CHCl}_{3}\right)$ afforded $\mathbf{6 a}(14 \mathrm{mg}, 98 \%)$ as a white solid. 
Analytical data for $\mathbf{6 a}$ :

${ }^{1}$ H NMR (600 MHz; $\left.\mathrm{CDCl}_{3}\right): \delta 7.30-7.27(\mathrm{~m}, 2 \mathrm{H}), 7.23(\mathrm{~d}, J=6.6 \mathrm{~Hz}, 2 \mathrm{H}), 7.20(\mathrm{t}, J=$ $7.2 \mathrm{~Hz}, 1 \mathrm{H}), 3.47(\mathrm{dd}, J=6.6,7.2 \mathrm{~Hz}, 2 \mathrm{H}), 3.29(\mathrm{t}, J=6.6 \mathrm{~Hz}, 2 \mathrm{H}), 2.99(\mathrm{dd}, J=7.2,8.4$ $\mathrm{Hz}, 2 \mathrm{H}), 2.56(\mathrm{dd}, J=7.8,8.4 \mathrm{~Hz}, 2 \mathrm{H}), 1.88(\mathrm{dt}, J=13,6.6 \mathrm{~Hz}, 2 \mathrm{H}), 1.82(\mathrm{dt}, J=14,7.8$ $\mathrm{Hz}, 2 \mathrm{H})$

${ }^{13}$ C NMR (150 MHz; $\left.\mathrm{CDCl}_{3}\right): \delta 170.7,141.5,128.41,128.37,126.0,46.5,45.6,36.7$, $31.2,26.0,24.3$

CAS Number: [151647-54-0]<smiles>O=C(Cc1ccccc1)Nc1ccccc1</smiles>

$N$-Benzyl-2-phenylacetamide (6b): Prepared according to the general procedure using MAC adduct 3b (22 mg, $0.10 \mathrm{mmol}), 1: 1 \mathrm{AcOH} / \mathrm{DME}(0.20 \mathrm{~mL})$, and $(R)-\mathrm{CSA}(26 \mathrm{mg}$, $0.11 \mathrm{mmol})$ for $5.5 \mathrm{~h}$, then anhydrous $\mathrm{CH}_{2} \mathrm{Cl}_{2}(0.20 \mathrm{~mL}), 1: 1$ benzylamine/ $\mathrm{CH}_{2} \mathrm{Cl}_{2}(0.46$ $\mathrm{mL}, 2.1 \mathrm{mmol}$ of pyrrolidine) and $\mathrm{Et}_{3} \mathrm{~N}(0.29 \mathrm{~mL}, 2.1 \mathrm{mmol})$. Purification by flash column chromatography $\left(\mathrm{SiO}_{2}, 67 \%\right.$ EtOAc/Hexanes) afforded $6 \mathbf{b}(23 \mathrm{mg}, 99 \%)$ as a white solid.

Analytical data for $\mathbf{6 b}$ :

${ }^{1} \mathbf{H}$ NMR $\left(600 \mathrm{MHz} ; \mathrm{CDCl}_{3}\right): \delta 7.35-7.33(\mathrm{~m}, 3 \mathrm{H}), 7.30-7.23(\mathrm{~m}, 5 \mathrm{H}), 7.17(\mathrm{~d}, J=7.2$ $\mathrm{Hz}, 2 \mathrm{H}), 4.40$ (d, $J=5.4 \mathrm{~Hz}, 2 \mathrm{H}), 3.62(\mathrm{~s}, 2 \mathrm{H})$

${ }^{13}$ C NMR $\left(150 \mathrm{MHz} ; \mathrm{CDCl}_{3}\right): \delta 170.9,138.1,134.7,129.4,129.0,128.6,127.43,127.38$, $127.35,43.8,43.5$

CAS Number: [7500-45-0]

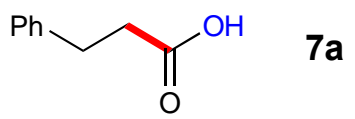

3-Phenylpropanoic acid (7a): Prepared according to the general procedure using MAC adduct 3a $(22 \mathrm{mg}, 0.10 \mathrm{mmol}), 1: 1 \mathrm{AcOH} / \mathrm{H}_{2} \mathrm{O}(0.48 \mathrm{~mL})$, and $(R)-\mathrm{CSA}(112 \mathrm{mg}, 0.48$ mmol) for $19 \mathrm{~h}$. Purification by flash column chromatography $\left(\mathrm{SiO}_{2}, 0 \rightarrow 1 \%\right.$ $\left.\mathrm{MeOH} / \mathrm{CHCl}_{3}\right)$ afforded $\mathbf{7 a}(14 \mathrm{mg}, 99 \%)$ as a white solid.

Analytical data for 7a:

${ }^{1} \mathbf{H}$ NMR $\left(600 \mathrm{MHz} ; \mathrm{CDCl}_{3}\right): \delta 11.51($ br s, $1 \mathrm{H}), 7.30(\mathrm{t}, J=7.8 \mathrm{~Hz}, 2 \mathrm{H}), 7.23-7.21(\mathrm{~m}$, $3 \mathrm{H}), 2.96(\mathrm{dd}, J=8.4,7.8 \mathrm{~Hz}, 2 \mathrm{H}), 2.69(\mathrm{dd}, J=8.4,7.2 \mathrm{~Hz}, 2 \mathrm{H})$

${ }^{13}$ C NMR (150 MHz; $\left.\mathrm{CDCl}_{3}\right): \delta 179.2,140.1,128.5,128.2,126.4,35.6,30.5$ 
CAS Number: [501-52-0]

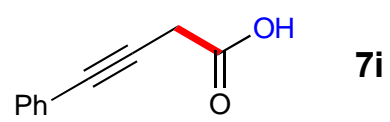

4-Phenylbut-3-ynoic acid (7i): Prepared according to the general procedure using MAC adduct $3 \mathbf{i}(25 \mathrm{mg}, 0.10 \mathrm{mmol}), 1: 1 \mathrm{AcOH} / \mathrm{H}_{2} \mathrm{O}(0.52 \mathrm{~mL})$, and $(R)$-CSA $(12 \mathrm{mg}, 0.051$ $\mathrm{mmol})$ for $15 \mathrm{~h}$. Purification by flash column chromatography $\left(\mathrm{SiO}_{2}, 5 \% \mathrm{MeOH} / \mathrm{CHCl}_{3}\right)$ afforded $7 \mathbf{i}$ (10.4 $\mathrm{mg}, 63 \%)$ as a pale yellow solid, and recovered starting material $\mathbf{3 i}$ (5.1 $\mathrm{mg}, 21 \%)$.

Analytical data for $\mathbf{7 i}$ :

${ }^{1} \mathbf{H}$ NMR $\left(600 \mathrm{MHz} ; \mathrm{CDCl}_{3}\right): \delta 7.46-7.44(\mathrm{~m}, 2 \mathrm{H}), 7.32-7.29(\mathrm{~m}, 3 \mathrm{H}), 3.59(\mathrm{~s}, 2 \mathrm{H})$

${ }^{13}$ C NMR (150 MHz; $\mathrm{CDCl}_{3}$ ): $\delta$ 173.9, 131.8 (x2), 128.4, 128.2 (x2), 122.6, 84.0, 80.1, 26.5

CAS Number: [7218-49-7]

\section{One-Pot Procedure for Conversion of Benzyl Alcohol to Homologated Amides}
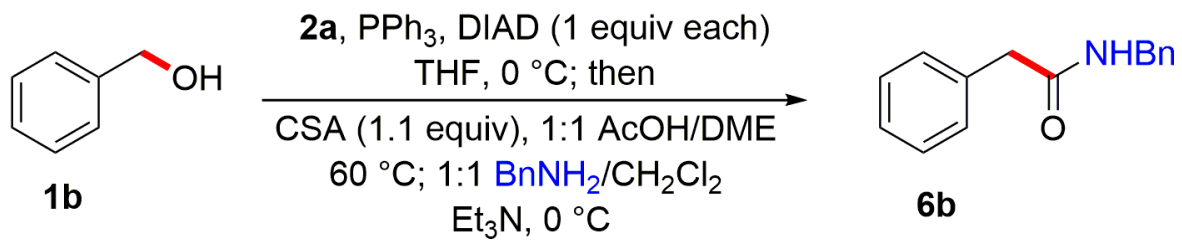

To an oven-dried two-neck round bottom flask was added a stir bar, $\mathrm{PPh}_{3}(48 \mathrm{mg}, 0.18$ mmol), and solvent $(2 \mathrm{~mL})$. The flask was capped with a septum and connected to $\mathrm{N}_{2}$ atmosphere. The mixture was cooled with an ice/water bath, and DIAD $(36 \mu \mathrm{L}, 0.18$ mmol) was added. The mixture was allowed to stir for $30 \mathrm{~min}$, then benzyl alcohol (1) $19 \mu \mathrm{L}, 0.18 \mathrm{mmol})$ was added. After an additional $30 \mathrm{~min}$, the MAC reagent $2 \mathbf{a}(23 \mathrm{mg}$, $0.18 \mathrm{mmol}$ ) was added. The ice/water bath was allowed to warm to ambient temperature, and the reaction was stirred for $15 \mathrm{~h}$ and concentrated in vacuo. To the residue was added 1:1 AcOH/DME $(0.36 \mathrm{~mL})$, and $(R)$-CSA $(46 \mathrm{mg}, 0.20 \mathrm{mmol})$. The flask was sealed with glass-caps and heated to $60{ }^{\circ} \mathrm{C}$ for $5.5 \mathrm{~h}$. The reaction mixture was cooled to ambient temperature, diluted with $\mathrm{CH}_{2} \mathrm{Cl}_{2} \quad(0.36 \mathrm{~mL})$, and cooled to $-40 \quad{ }^{\circ} \mathrm{C}$. $1: 1$ Benzylamine/ $\mathrm{CH}_{2} \mathrm{Cl}_{2}\left(0.81 \mathrm{~mL}, 3.7 \mathrm{mmol}\right.$ of $\left.\mathrm{BnNH}_{2}\right)$ was added dropwise over $10 \mathrm{~min}$, then $\mathrm{Et}_{3} \mathrm{~N}(0.52 \mathrm{~mL}, 3.7 \mathrm{mmol})$ was added. After $5 \mathrm{~min}$, the reaction was warmed to $0{ }^{\circ} \mathrm{C}$. After 1 hour at $0{ }^{\circ} \mathrm{C}$, the reaction was slowly quenched with sat. aq. $\mathrm{NH}_{4} \mathrm{Cl}(5 \mathrm{~mL})$. The mixture was extracted three times with $\mathrm{CH}_{2} \mathrm{Cl}_{2}$ and the combined organics were dried over anh. $\mathrm{Na}_{2} \mathrm{SO}_{4}$ and concentrated to give a crude solid. The crude solid was diluted with EtOAc, filtered through a cotton plug, and concentrated to give a crude oil. 
Purification by flash column chromatography $\left(\mathrm{SiO}_{2}, 50 \%\right.$ EtOAc/Hexanes, then 1:2:1 EtOAc/Hexanes/ $\mathrm{CH}_{2} \mathrm{Cl}_{2}$, then $\left.1.0 \% \mathrm{MeOH} / \mathrm{CHCl}_{3}\right)$ afforded $\mathbf{6 b}(39 \mathrm{mg}, 96 \%$ ).<smiles>OCc1ccccc1</smiles>

$1 \mathrm{~b}$ 2a, $\mathrm{PPh}_{3}, \mathrm{DIAD}(1$ equiv each)

$$
\begin{aligned}
& \text { THF, } 0{ }^{\circ} \mathrm{C} \text {; then } \\
& \mathrm{CSA} \text { (1.1 equiv), 1:1 AcOH/DME } \\
& 60^{\circ} \mathrm{C} ; \mathrm{CH}_{2} \mathrm{Cl}_{2}, 2.0 \mathrm{M} \mathrm{EtNH}_{2} / \mathrm{THF} \\
& \mathrm{Et}_{3} \mathrm{~N}, 0^{\circ} \mathrm{C}
\end{aligned}
$$

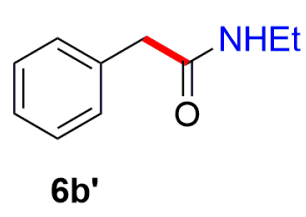

To an oven-dried two-neck round bottom flask was added a stir bar, $\mathrm{PPh}_{3}(34 \mathrm{mg}, 0.13$ $\mathrm{mmol}$ ), and solvent $(1 \mathrm{~mL})$. The flask was capped with a septum and connected to a $\mathrm{N}_{2}$ atmosphere. The mixture was cooled with an ice-water bath, and a solution of DMEAD (30 $\mathrm{mg}, 0.13 \mathrm{mmol})$ in THF $(0.5 \mathrm{~mL})$ was added using a cannula. The mixture was allowed to stir for $30 \mathrm{~min}$, then benzyl alcohol $\mathbf{1 b}(13 \mu \mathrm{L}, 0.13 \mathrm{mmol})$ was added. After an additional $30 \mathrm{~min}$, the MAC reagent $\mathbf{2 a}(16 \mathrm{mg}, 0.13 \mathrm{mmol})$ was added. The ice/water bath was allowed to warm to ambient temperature, and the reaction was stirred for $18 \mathrm{~h}$ and concentrated in vacuo. To the residue was added 1:1 AcOH/DME $(0.26 \mathrm{~mL})$, and $(R)$-CSA (33 mg, $0.14 \mathrm{mmol})$. The flask was sealed with glass stoppers and heated to 60 ${ }^{\circ} \mathrm{C}$ for $5 \mathrm{~h}$. The reaction mixture was cooled to ambient temperature, diluted with $\mathrm{CH}_{2} \mathrm{Cl}_{2}$ $(0.26 \mathrm{~mL})$, and cooled to $-40{ }^{\circ} \mathrm{C}$. A $2.0 \mathrm{M}$ solution of ethylamine in THF $(1.3 \mathrm{~mL}, 2.6$ mmol of $\left.\mathrm{EtNH}_{2}\right)$ was added dropwise over $10 \mathrm{~min}$, then $\mathrm{Et}_{3} \mathrm{~N}(0.36 \mathrm{~mL}, 2.6 \mathrm{mmol})$ was added. After $5 \mathrm{~min}$, the reaction was warmed to $0{ }^{\circ} \mathrm{C}$. After $1.5 \mathrm{~h}$ at $0{ }^{\circ} \mathrm{C}$, the reaction was slowly quenched with sat. aq. $\mathrm{NH}_{4} \mathrm{Cl}(5 \mathrm{~mL})$. The mixture was extracted three times with $\mathrm{CH}_{2} \mathrm{Cl}_{2}$ and the combined organics were dried over anh. $\mathrm{Na}_{2} \mathrm{SO}_{4}$ and concentrated to give a crude solid. The crude solid was washed with $\mathrm{H}_{2} \mathrm{O}$, and dried over anh. $\mathrm{Na}_{2} \mathrm{SO}_{4}$ and concentrated to give a pink crude solid. Purification by flash column chromatography $\left(\mathrm{SiO}_{2}, 67 \%\right.$ EtOAc/Hexanes) afforded 6b' (18 mg, 85\%).

Analytical data for $\mathbf{6} \mathbf{b}^{\prime}$ :

${ }^{1} \mathbf{H}$ NMR $\left(600 \mathrm{MHz} ; \mathrm{CDCl}_{3}\right): \delta 7.36(\mathrm{dd}, J=8.4,7.2 \mathrm{~Hz}, 2 \mathrm{H}), 7.30(\mathrm{dd}, J=7.2,5.4 \mathrm{~Hz}$, $1 \mathrm{H}), 7.26(\mathrm{~d}, J=9.0 \mathrm{~Hz}, 1 \mathrm{H}), 7.26(\mathrm{~d}, J=7.2 \mathrm{~Hz}, 1 \mathrm{H}), 3.57(\mathrm{~s}, 3 \mathrm{H}), 3.25$ (ddd, $J=15$, $7.85 .4 \mathrm{~Hz}, 2 \mathrm{H}), 1.06(\mathrm{t}, J=7.2 \mathrm{~Hz}, 3 \mathrm{H})$

${ }^{13}$ C NMR (150 MHz; $\left.\mathrm{CDCl}_{3}\right): \delta 170.8,134.9,129.5,129.0,127.3,43.8,34.5,14.7$

CAS Number: [5465-00-9] 


\section{Selected NMR Spectra}
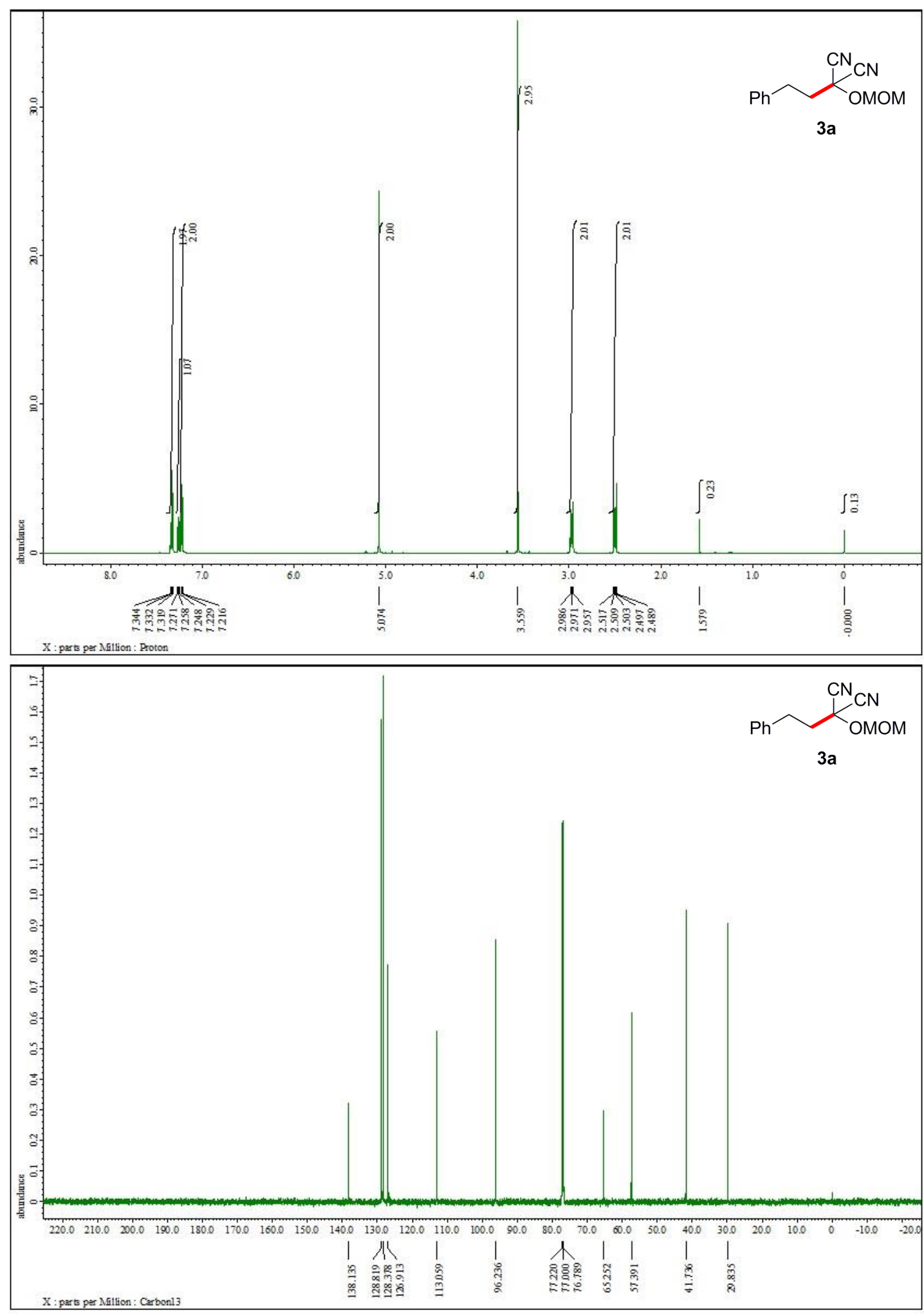
Supporting Information

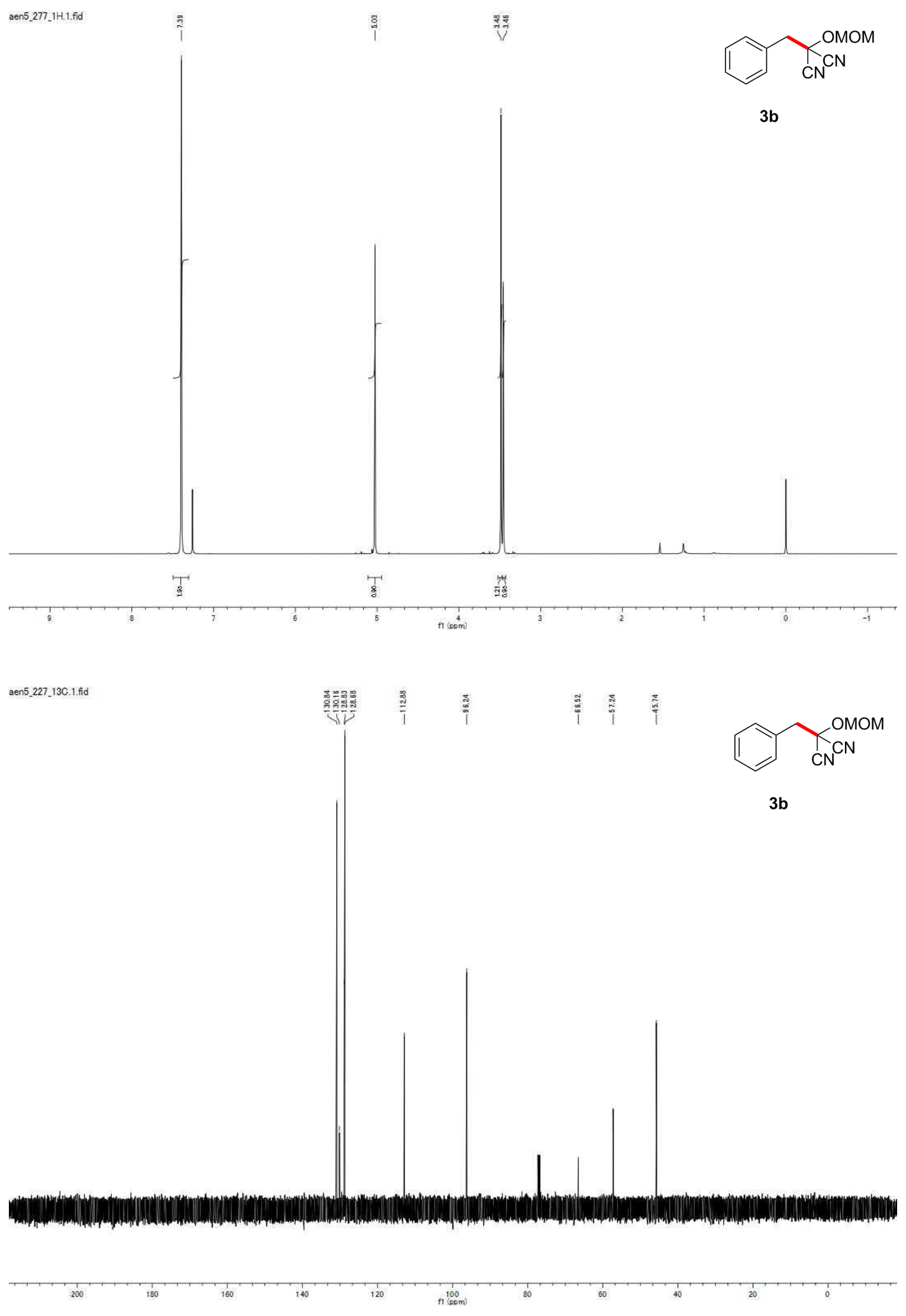


Supporting Information
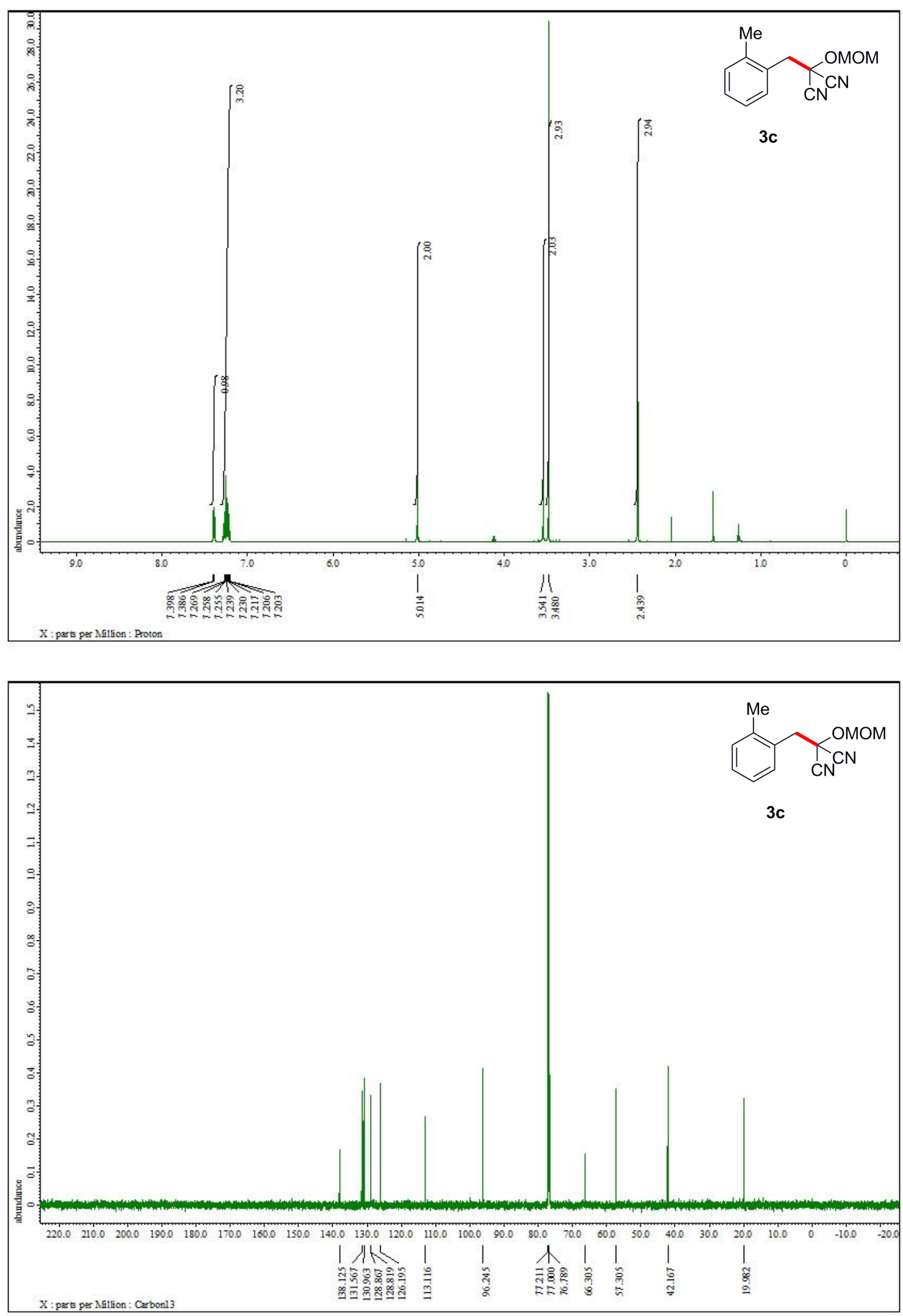
Supporting Information
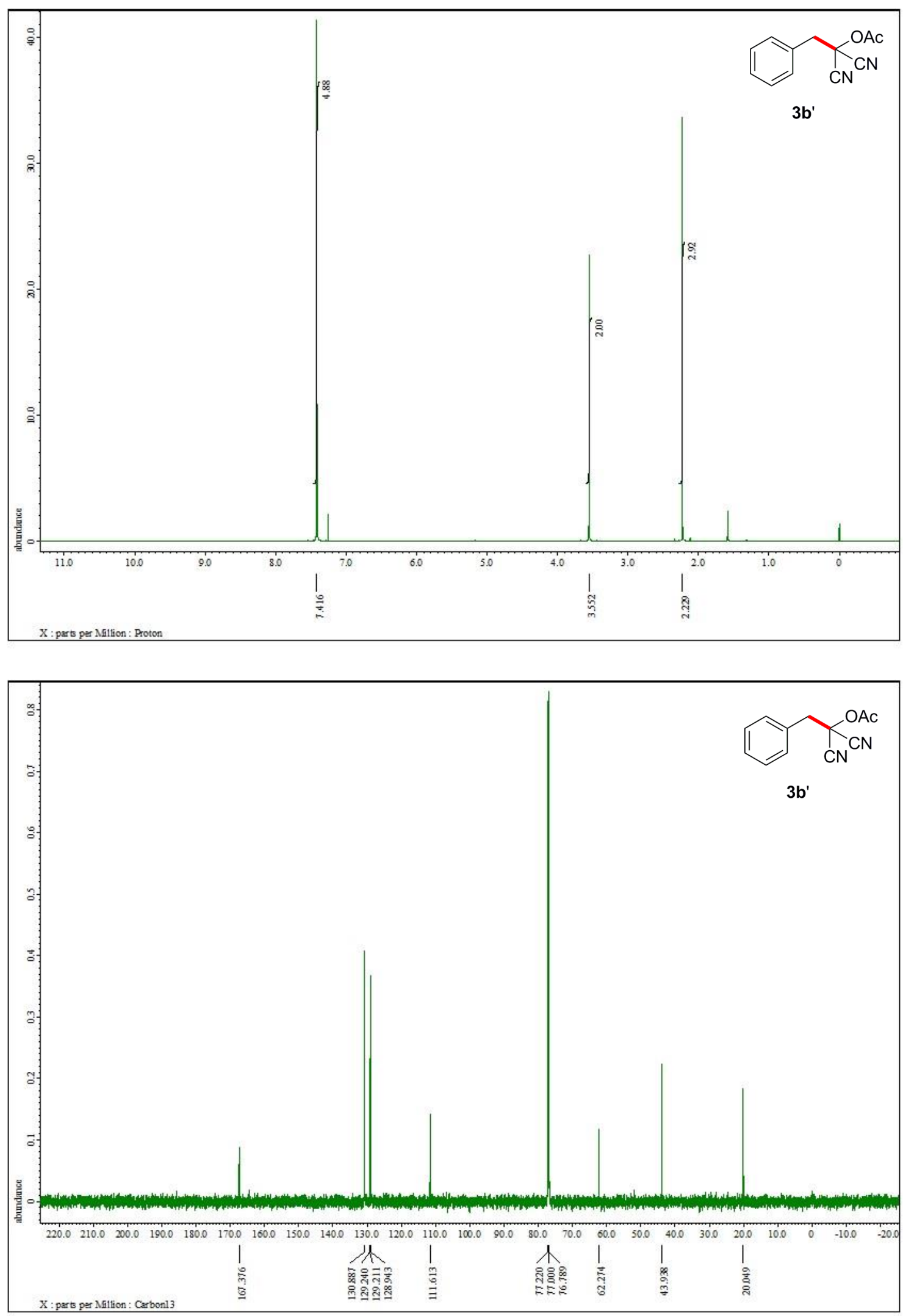

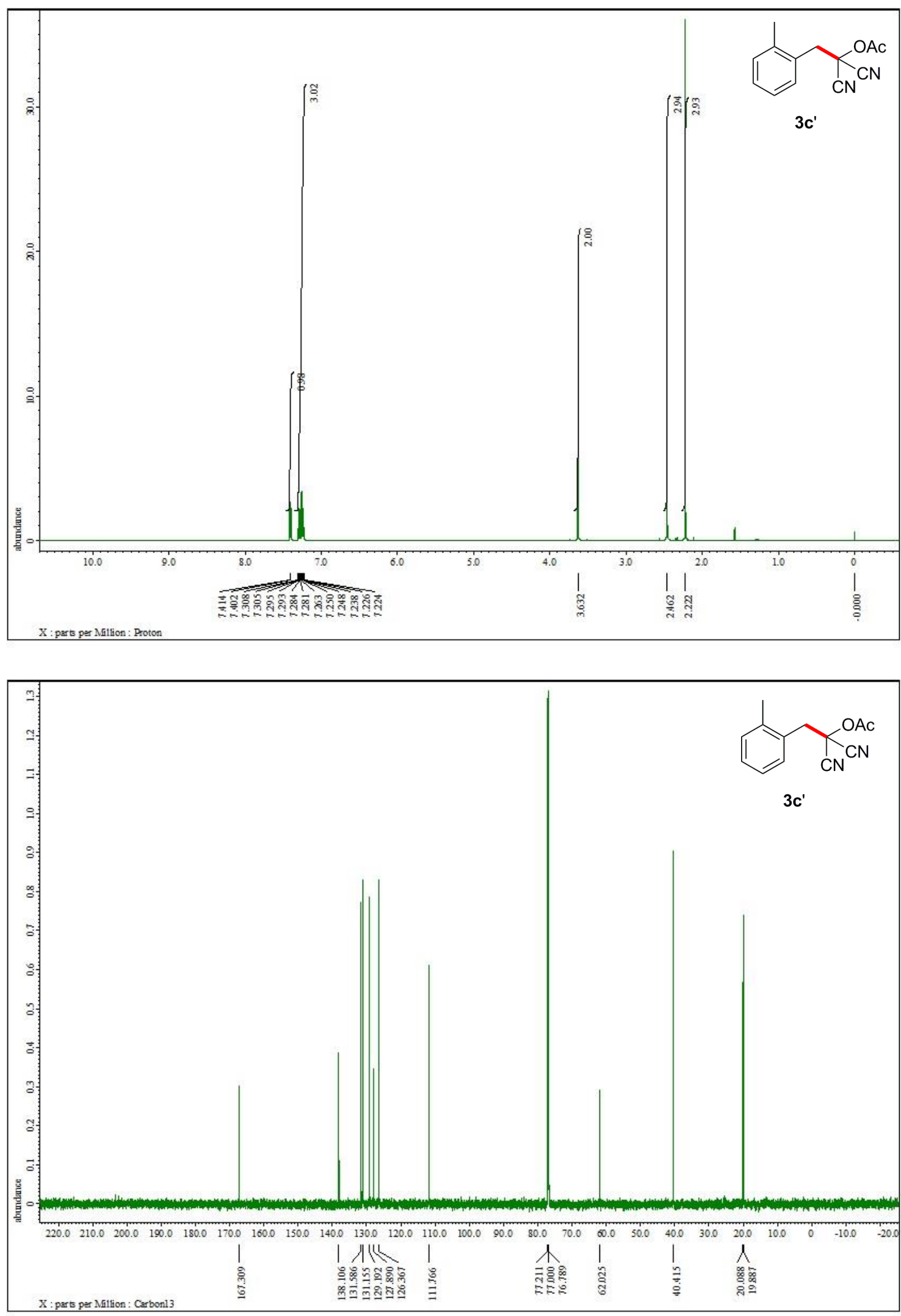
Supporting Information

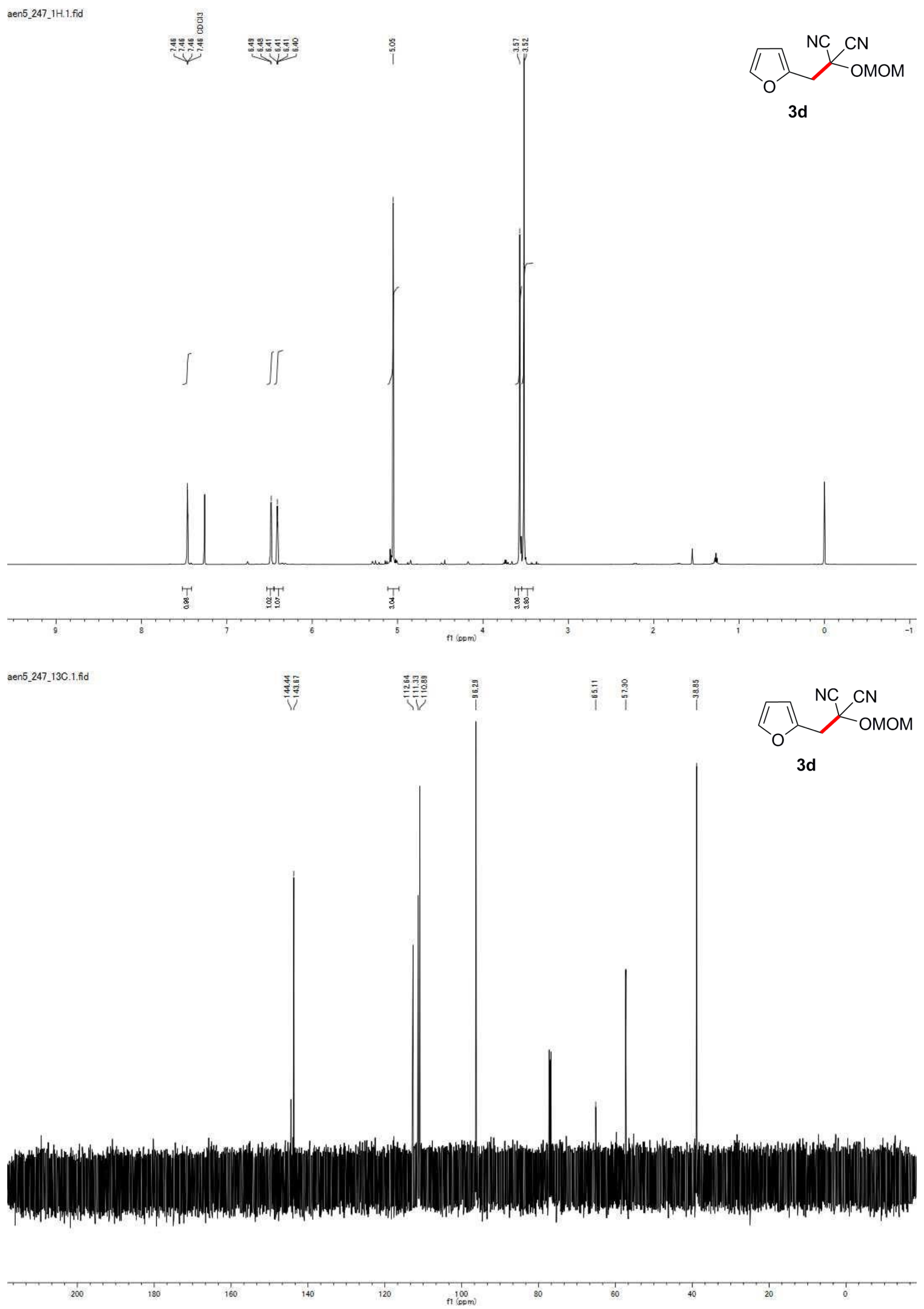


Supporting Information
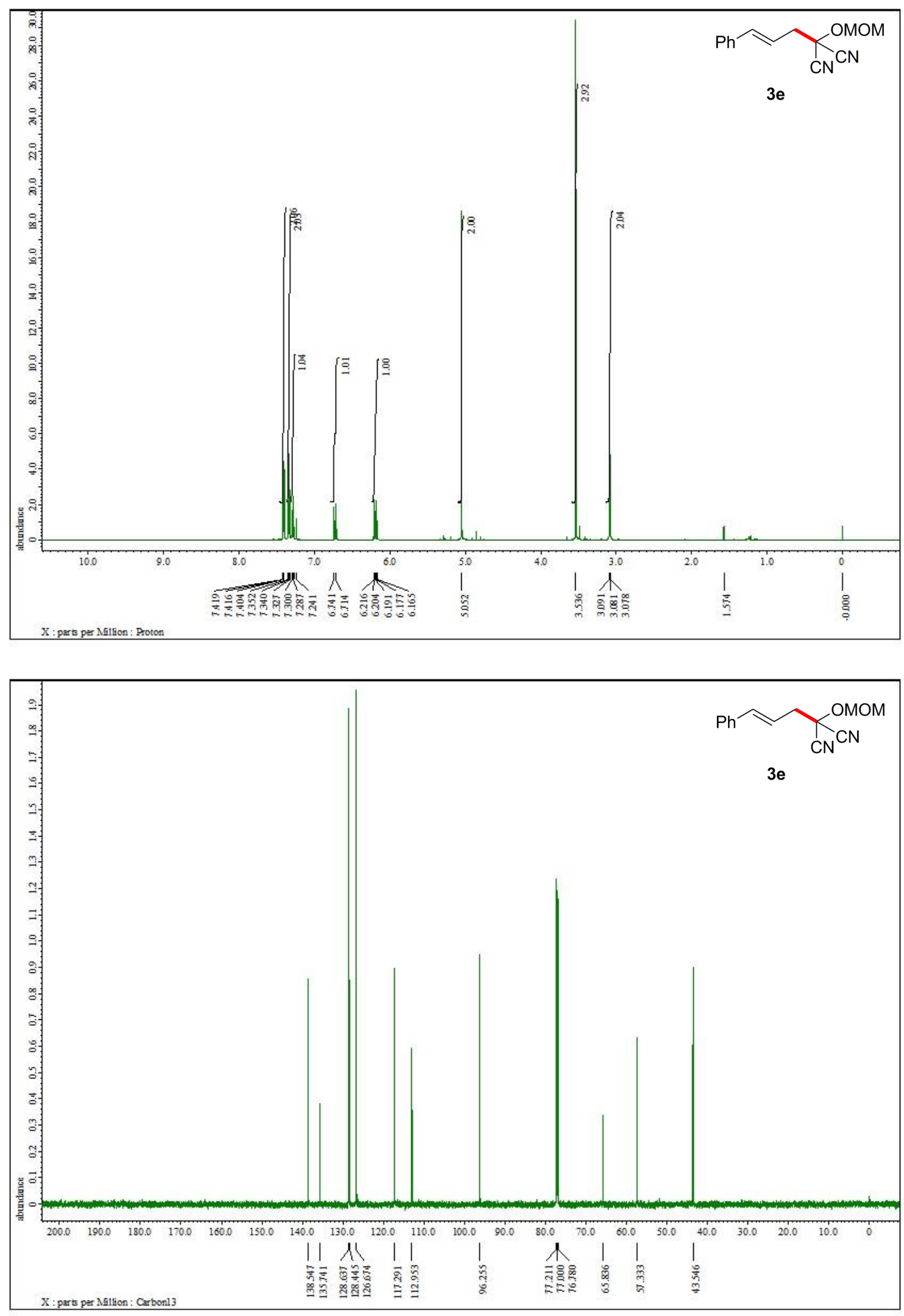
Supporting Information
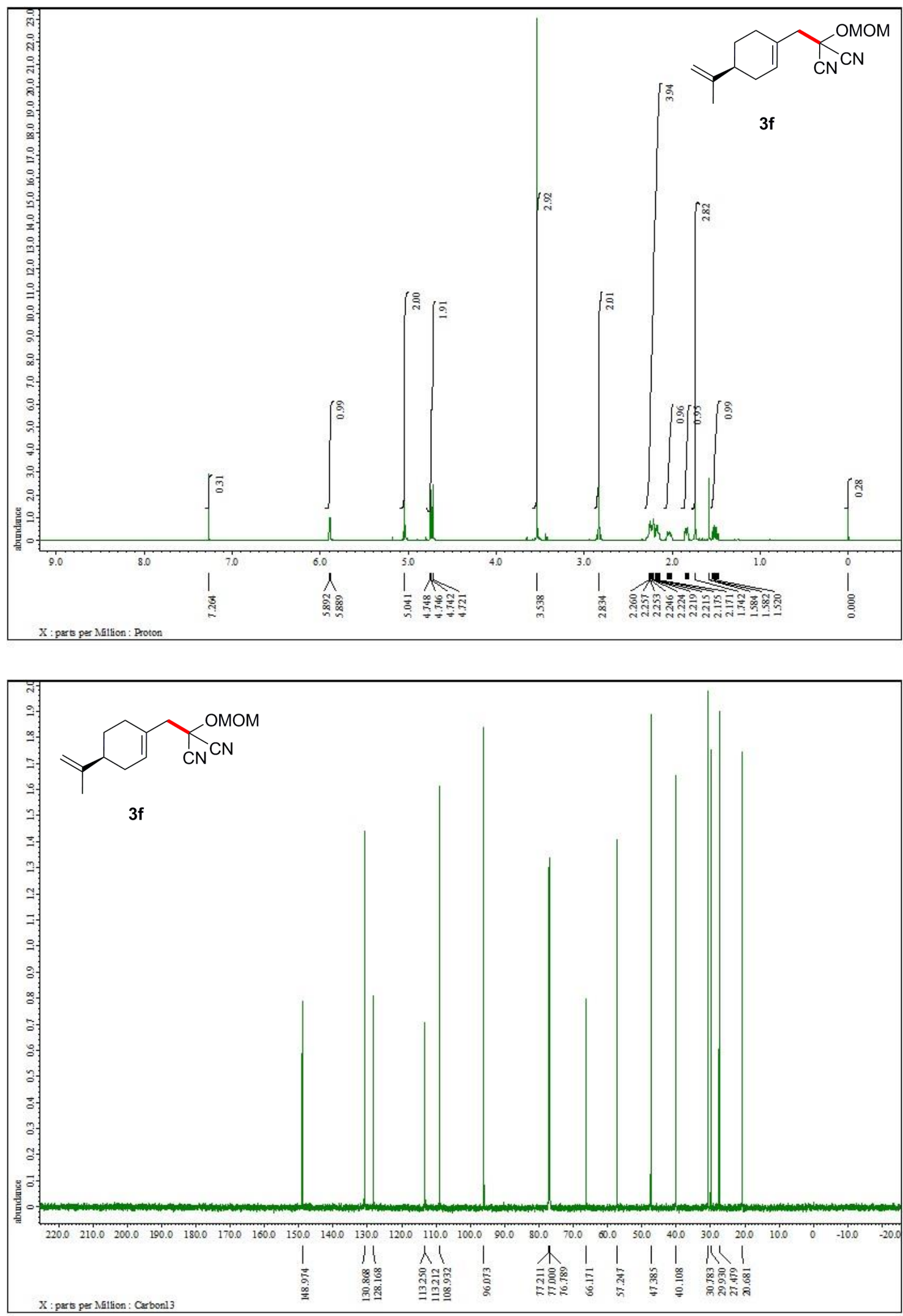
Supporting Information
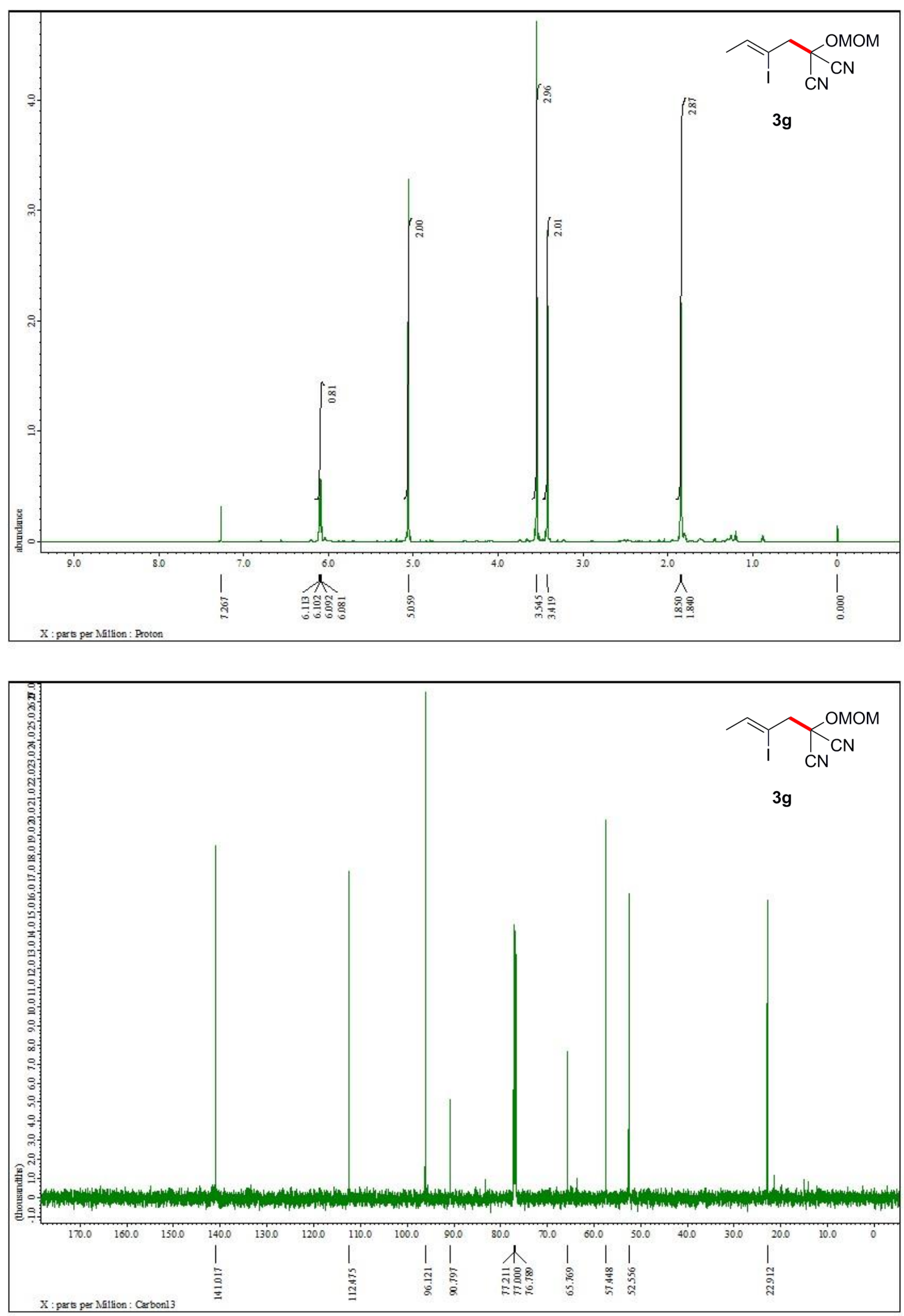


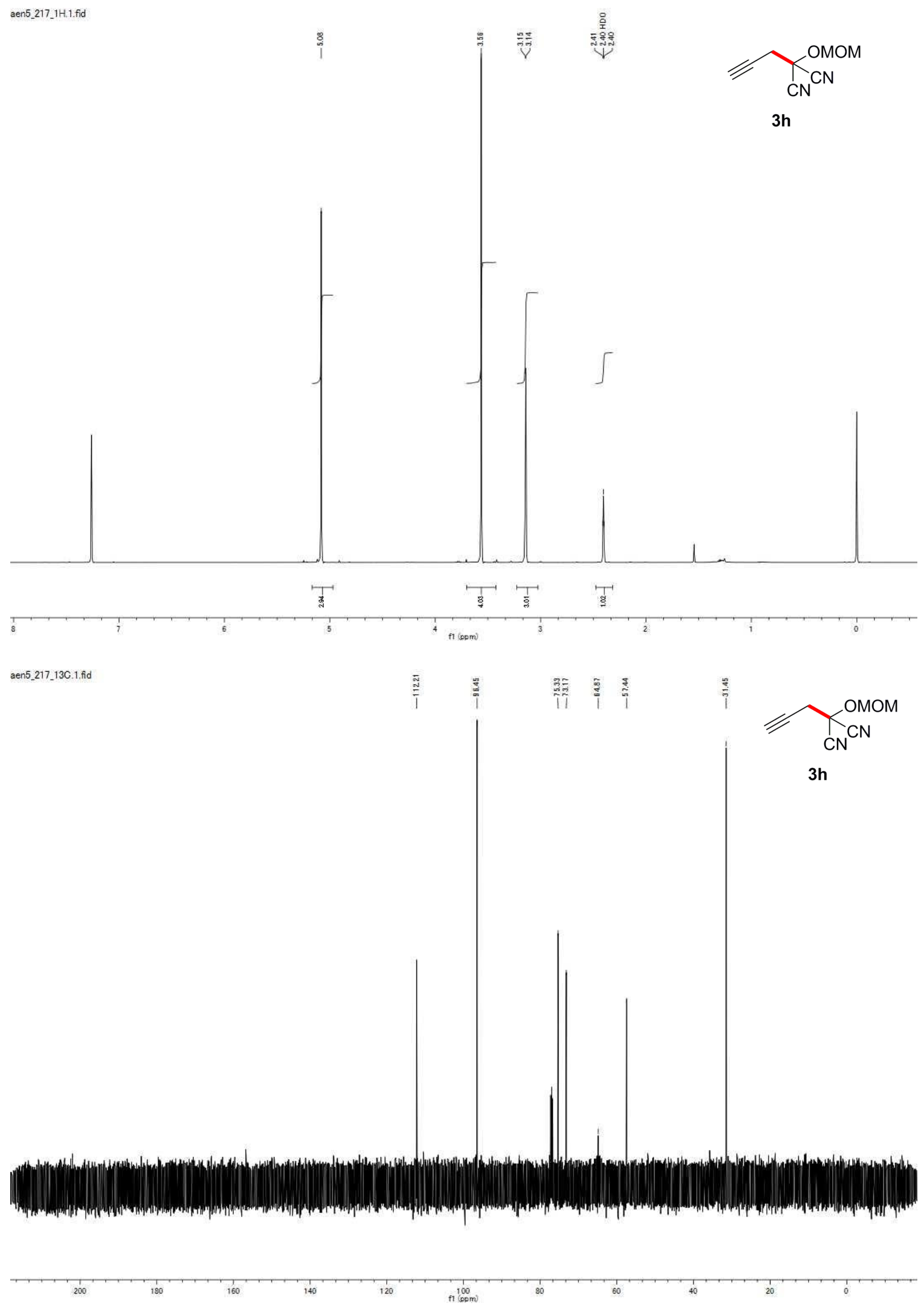


Supporting Information

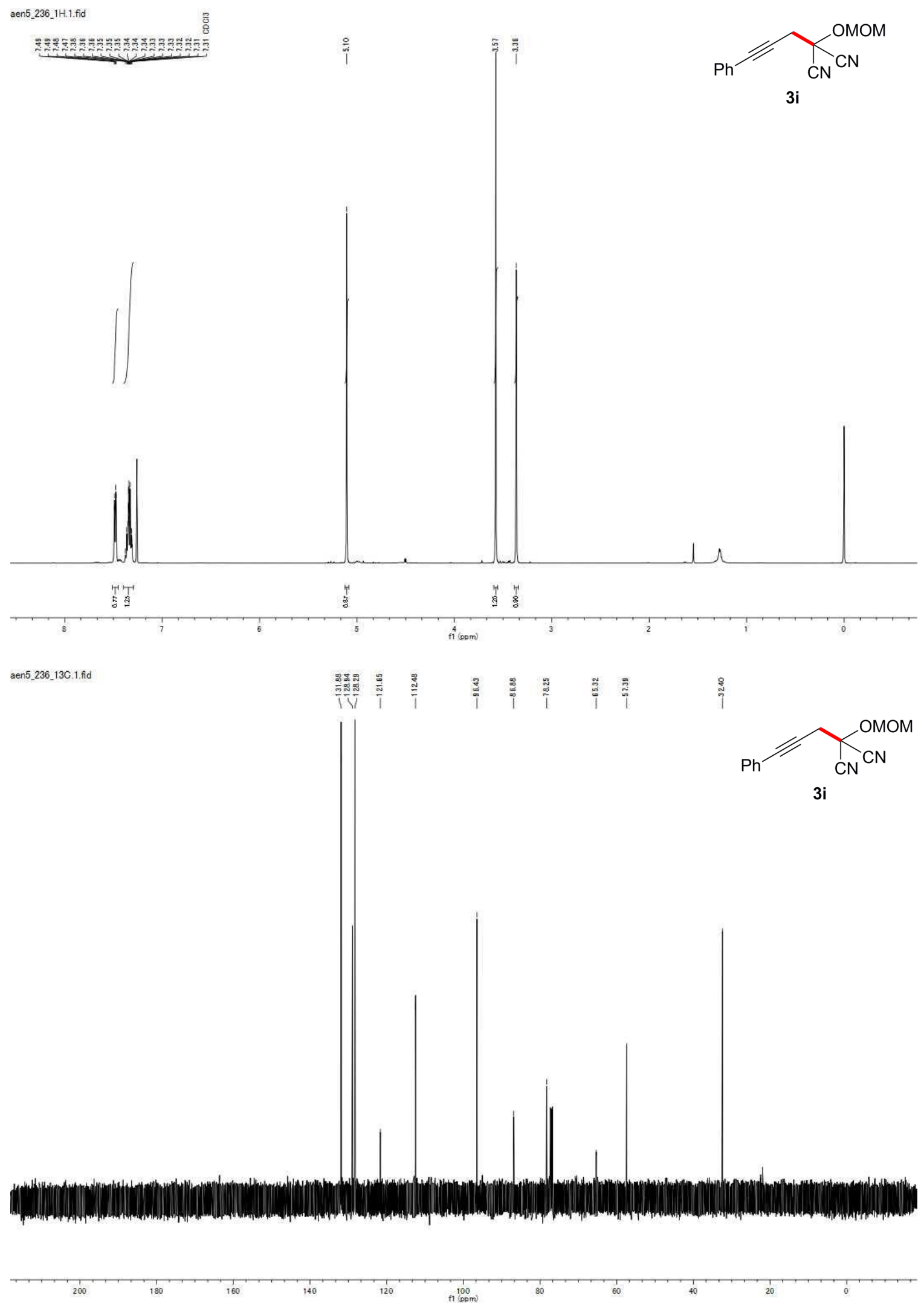


Supporting Information
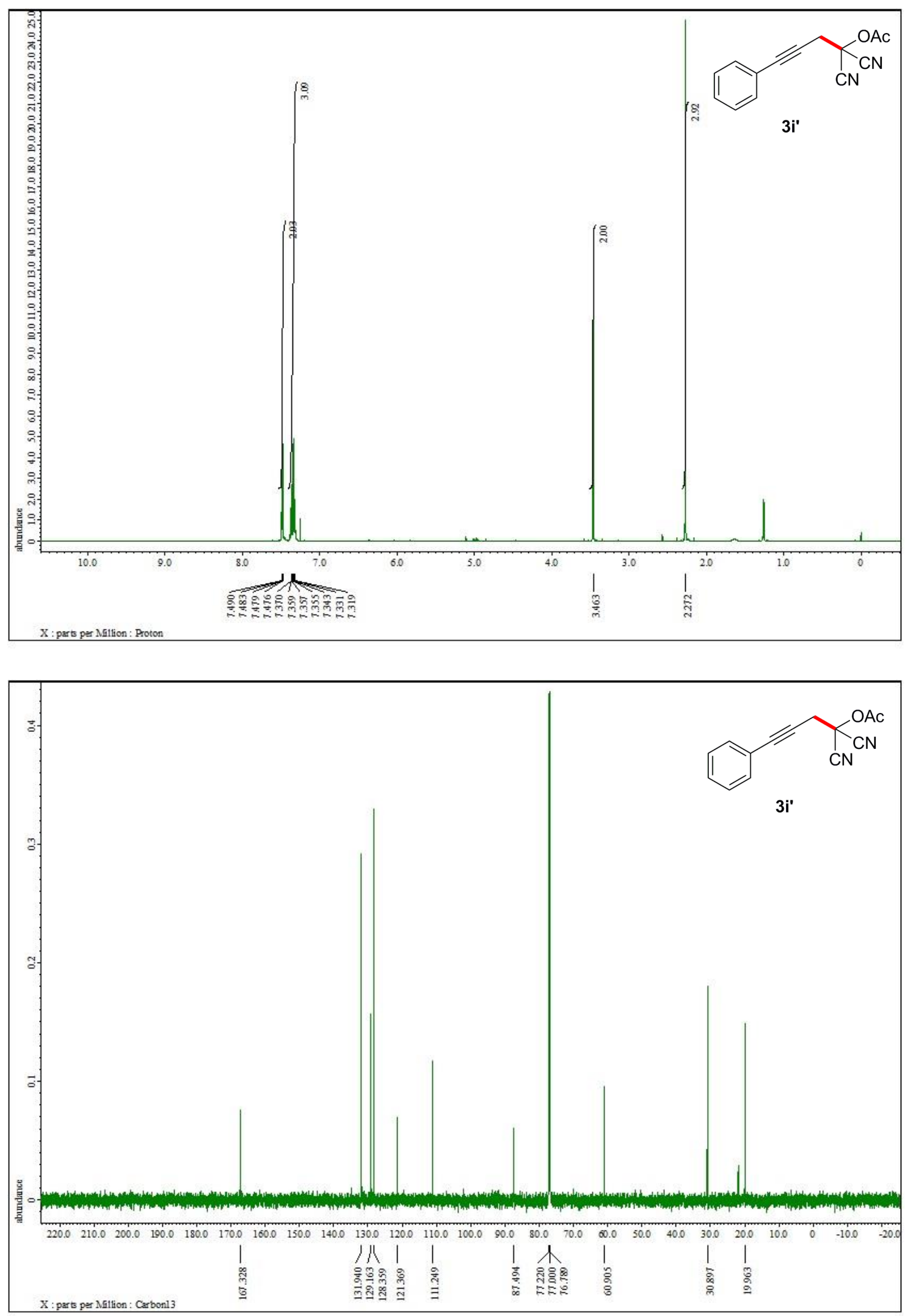

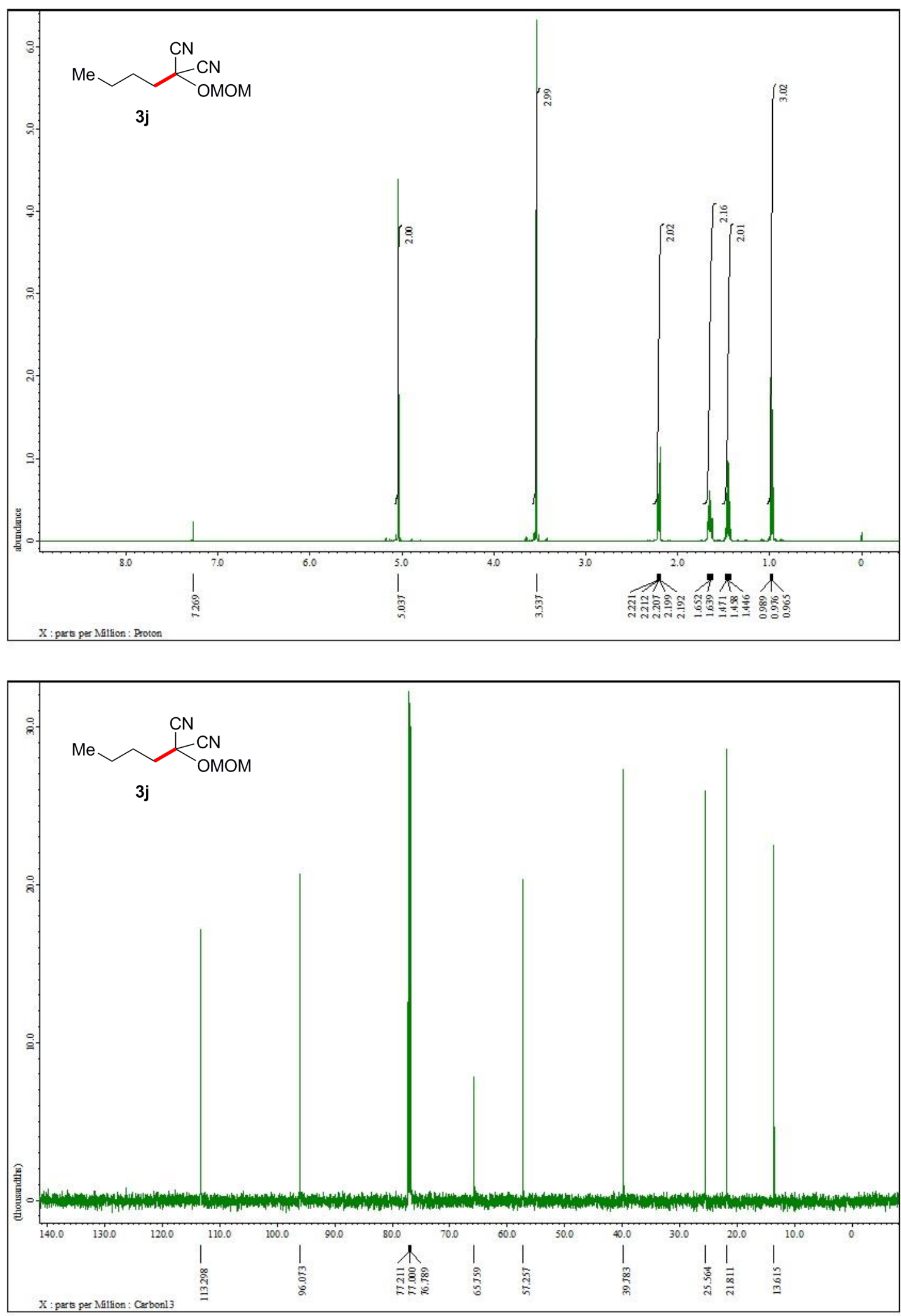
Supporting Information

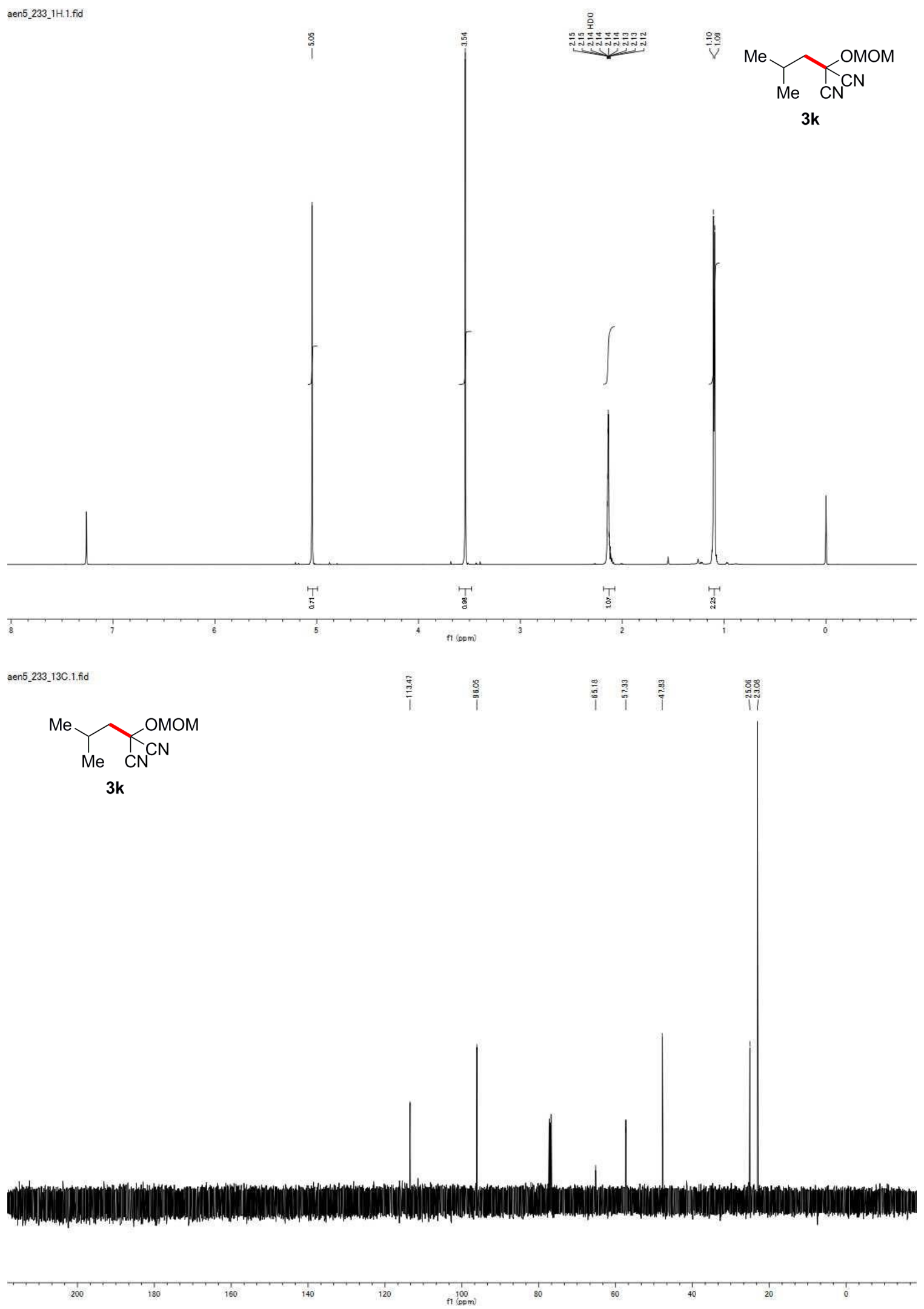



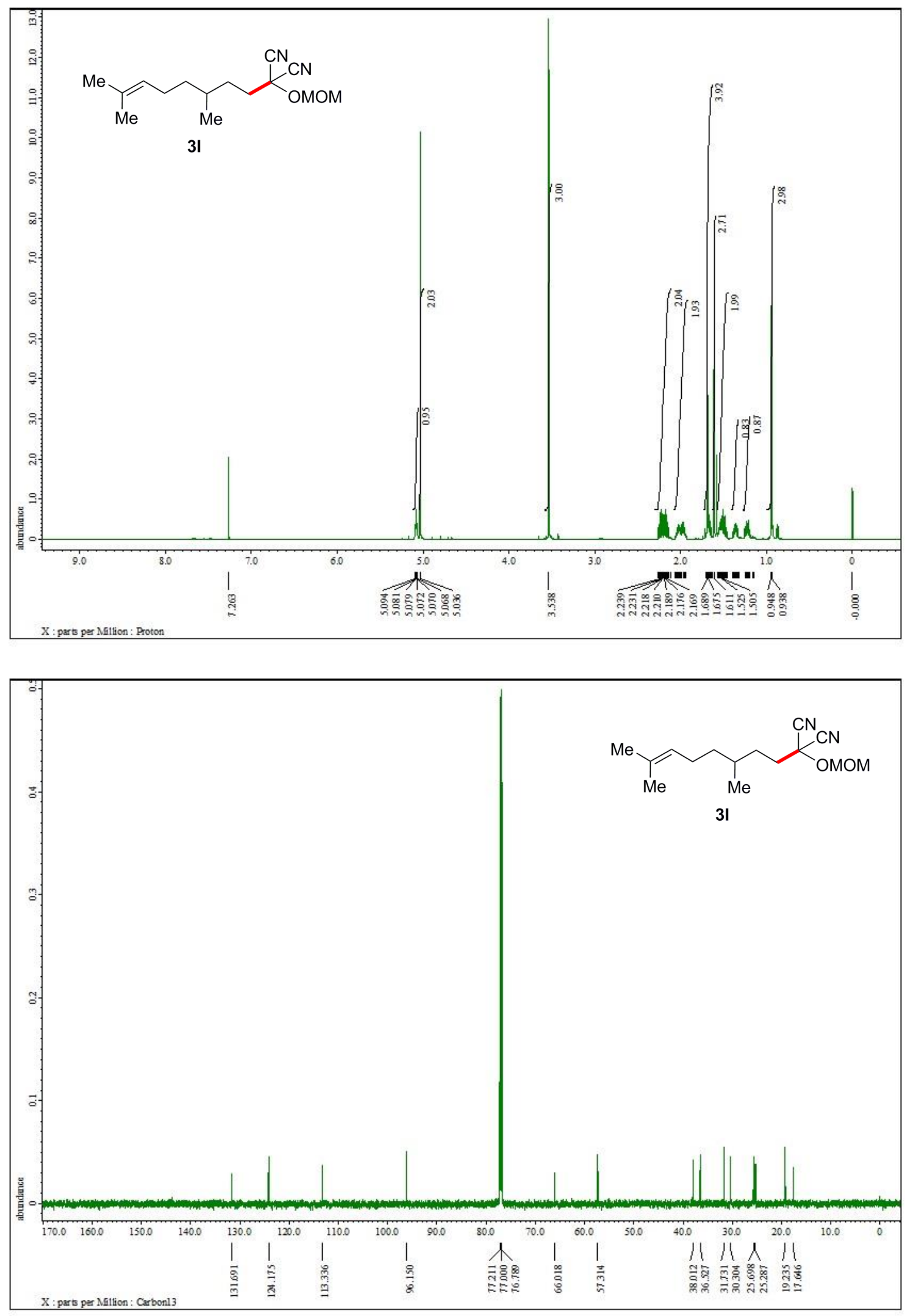

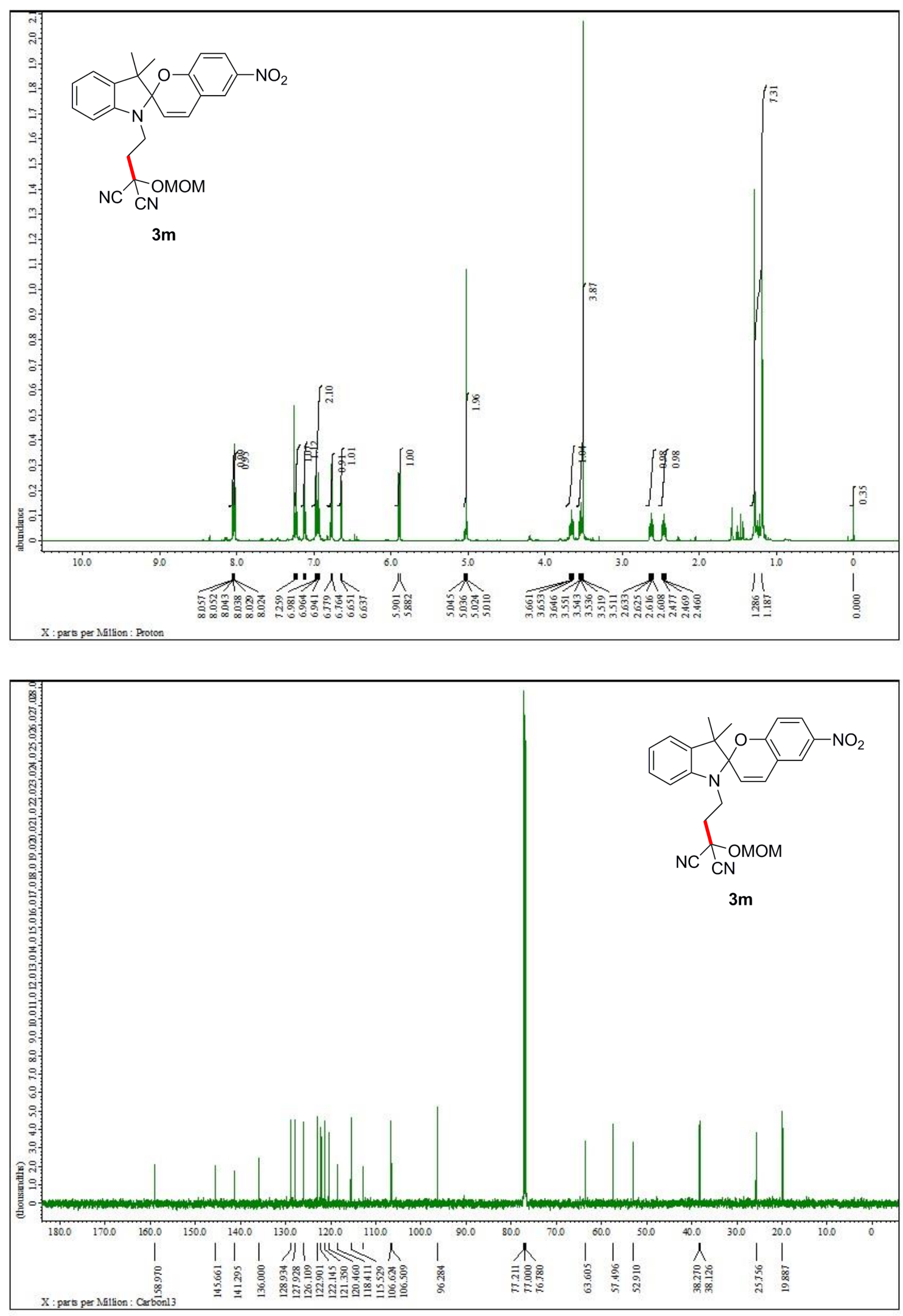

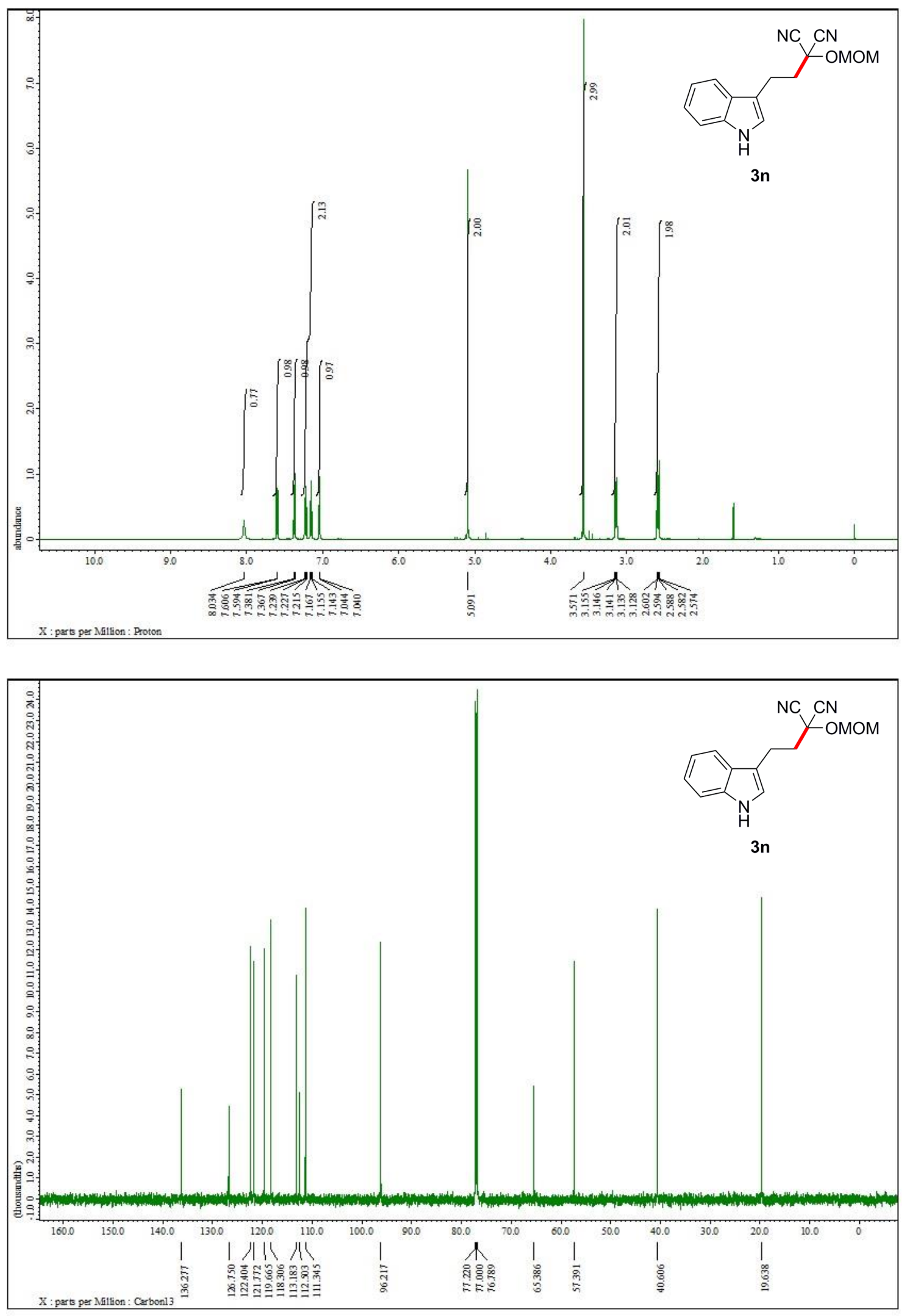

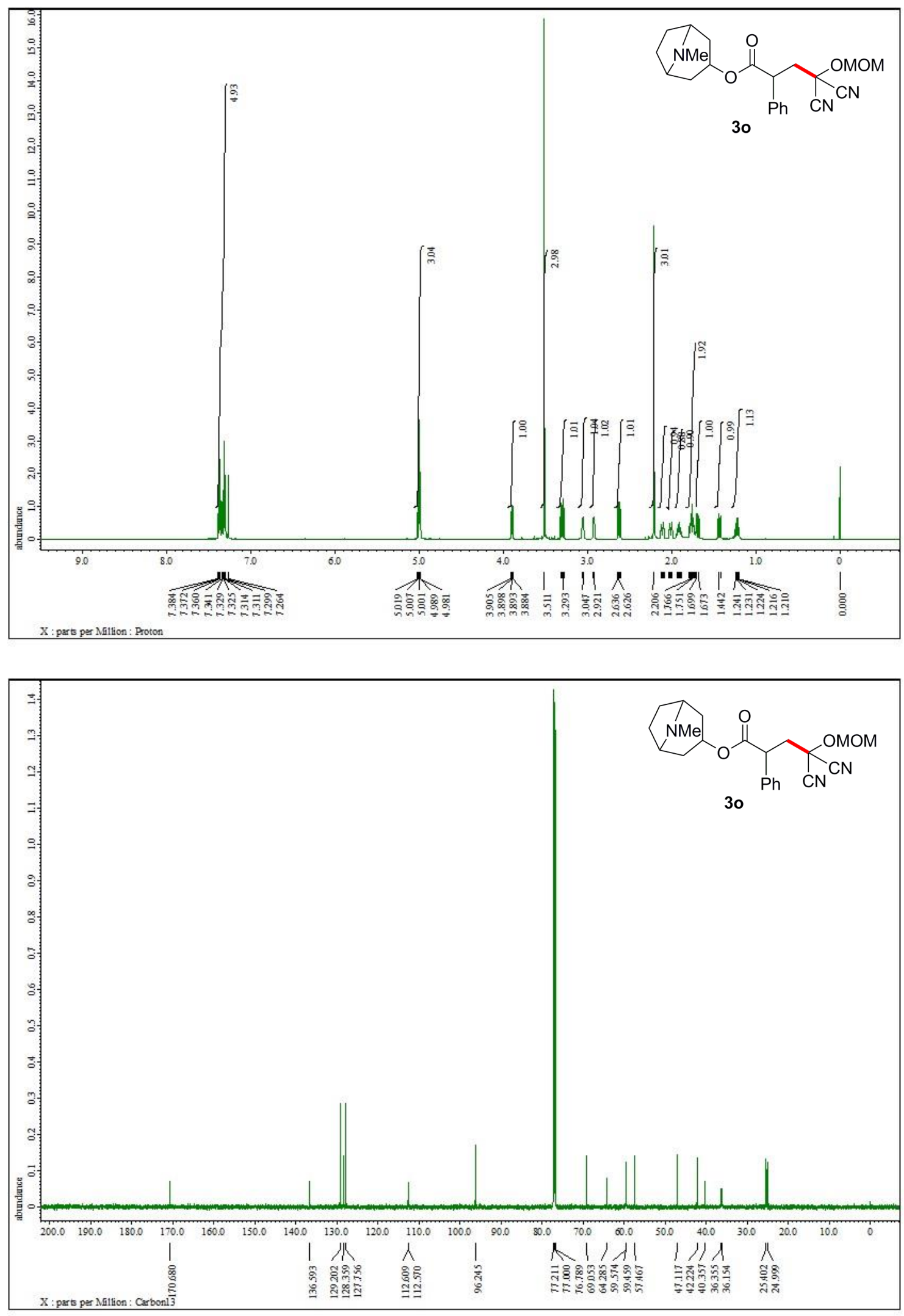


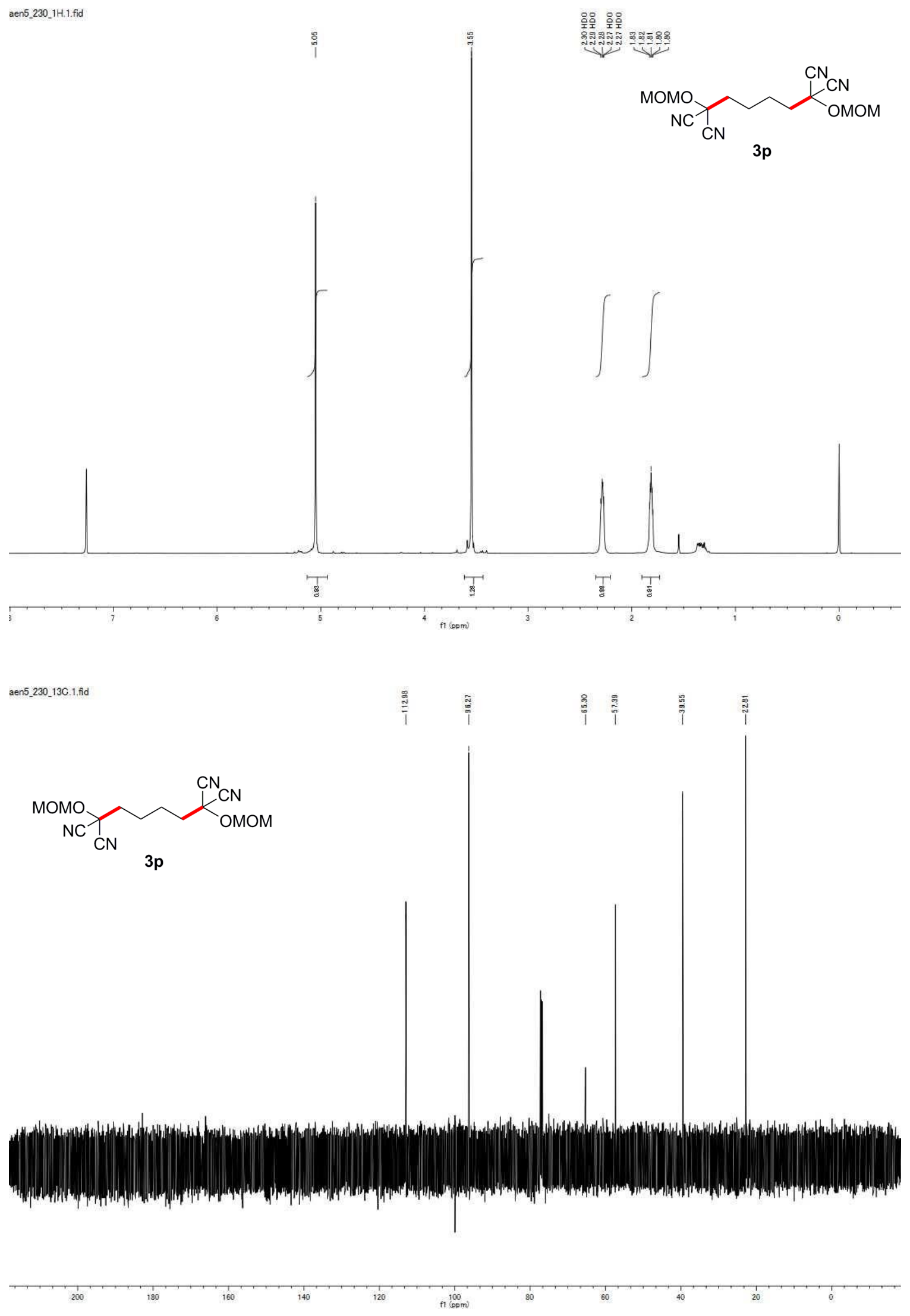



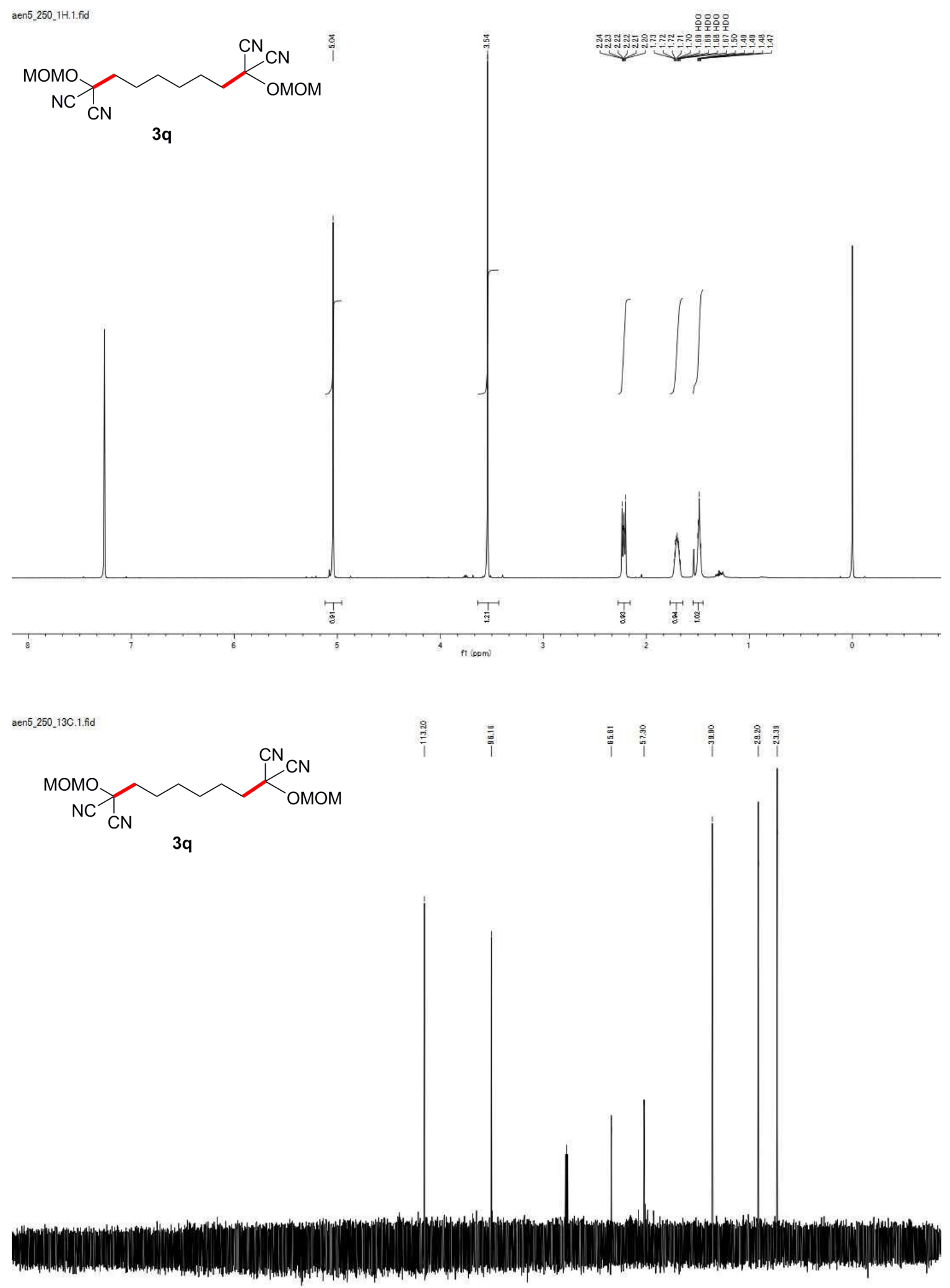

200

160

140

120

$\mathrm{fl}_{10 \mathrm{~mm})}^{100}$ 

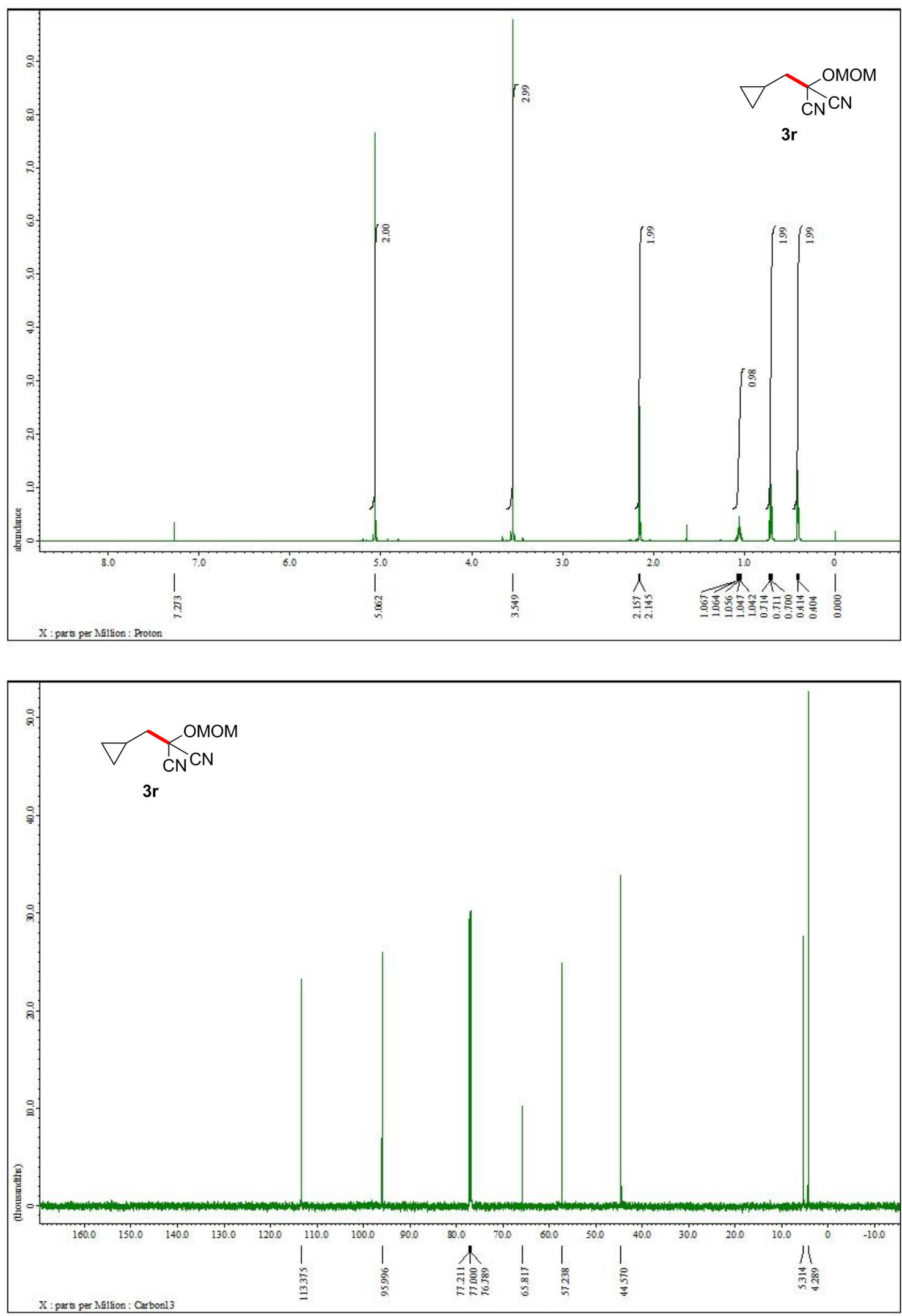

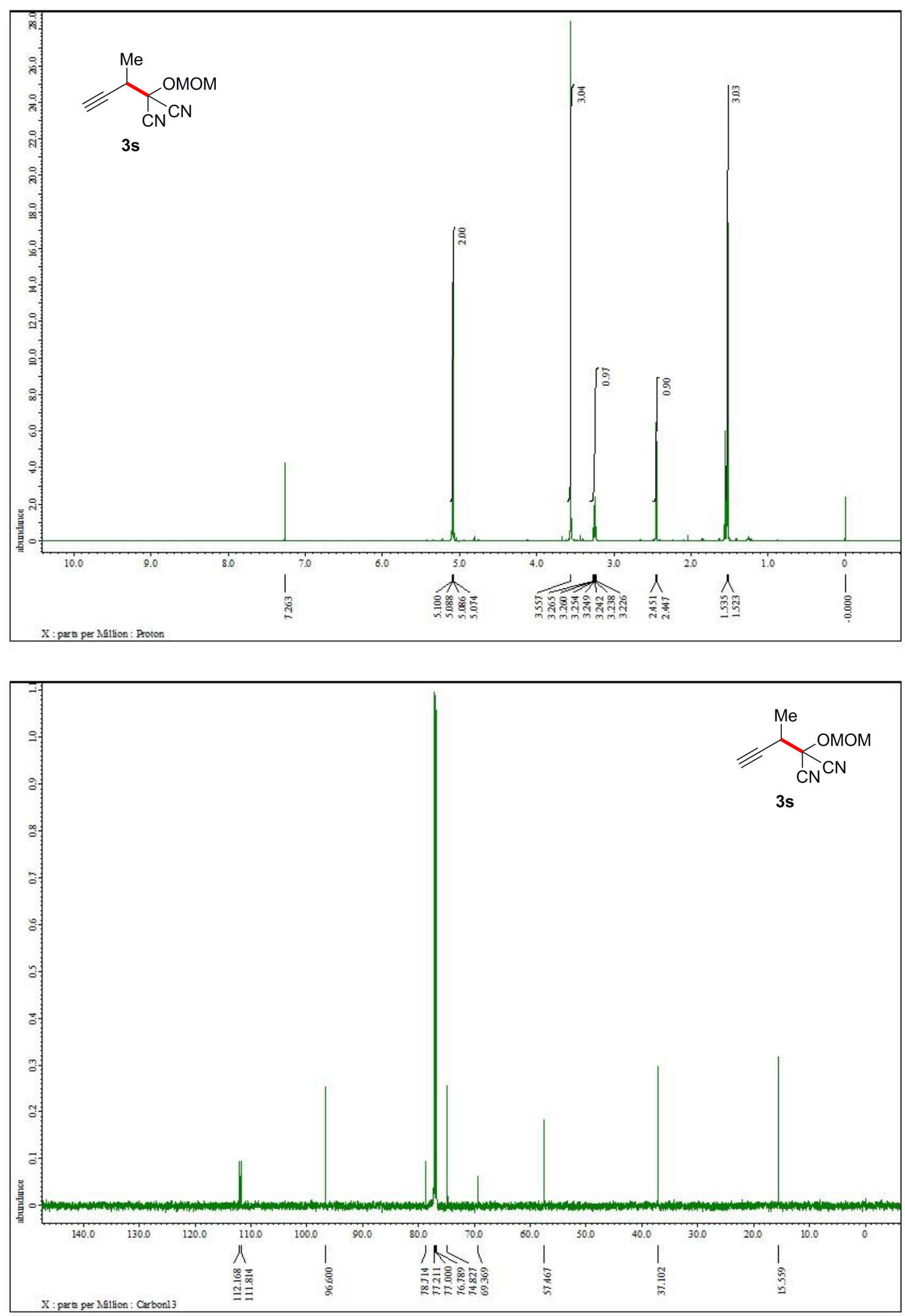
Supporting Information
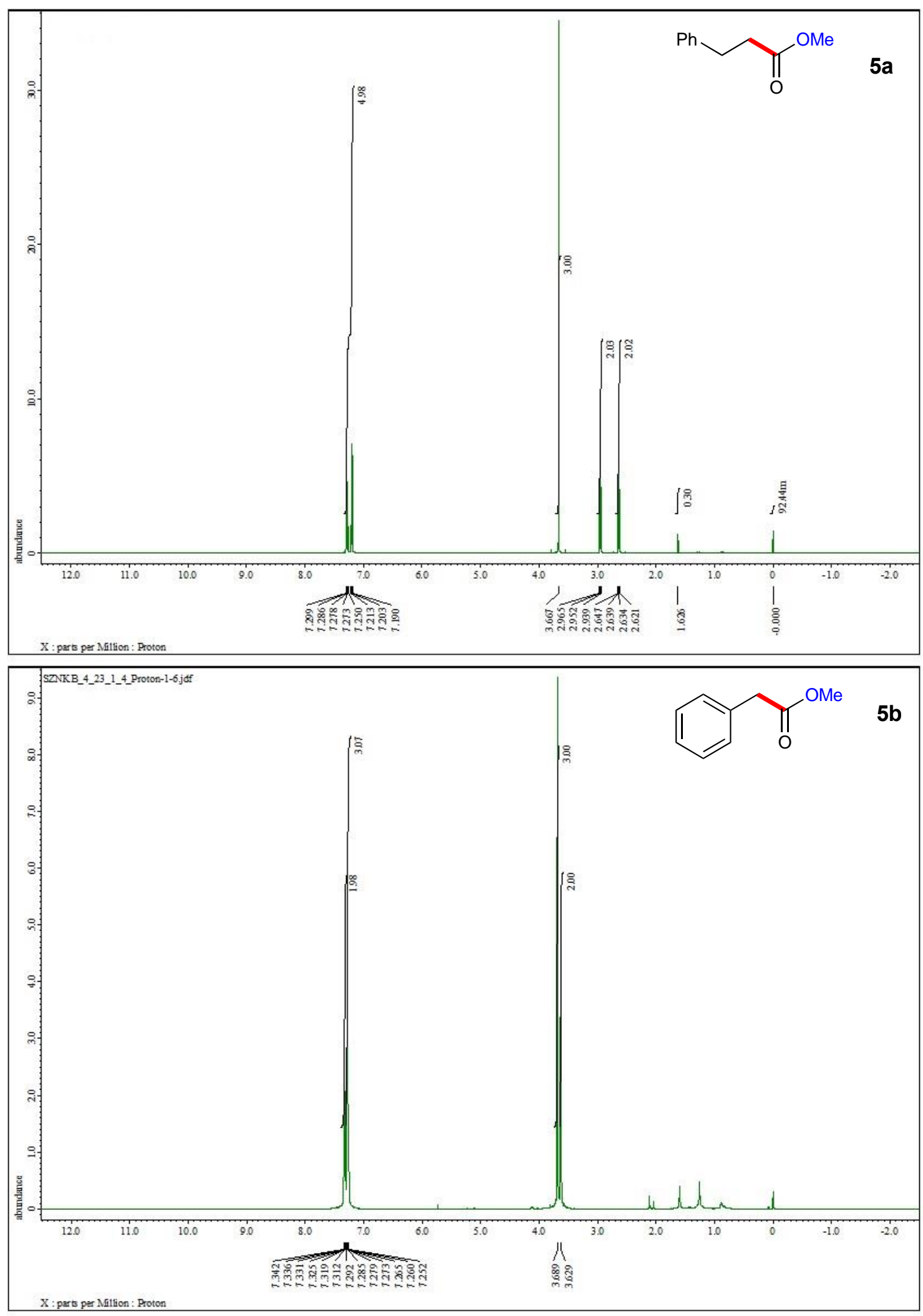

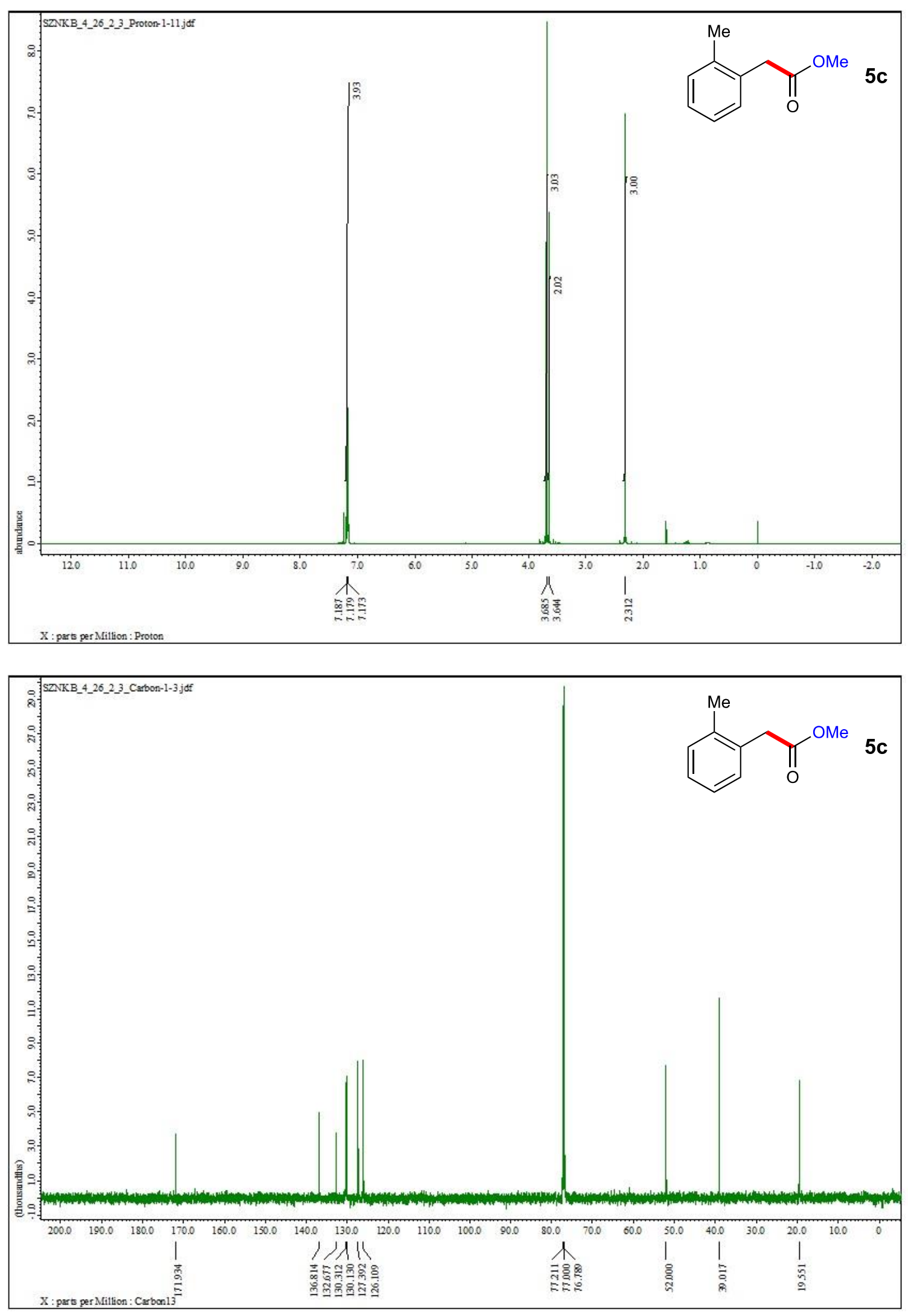

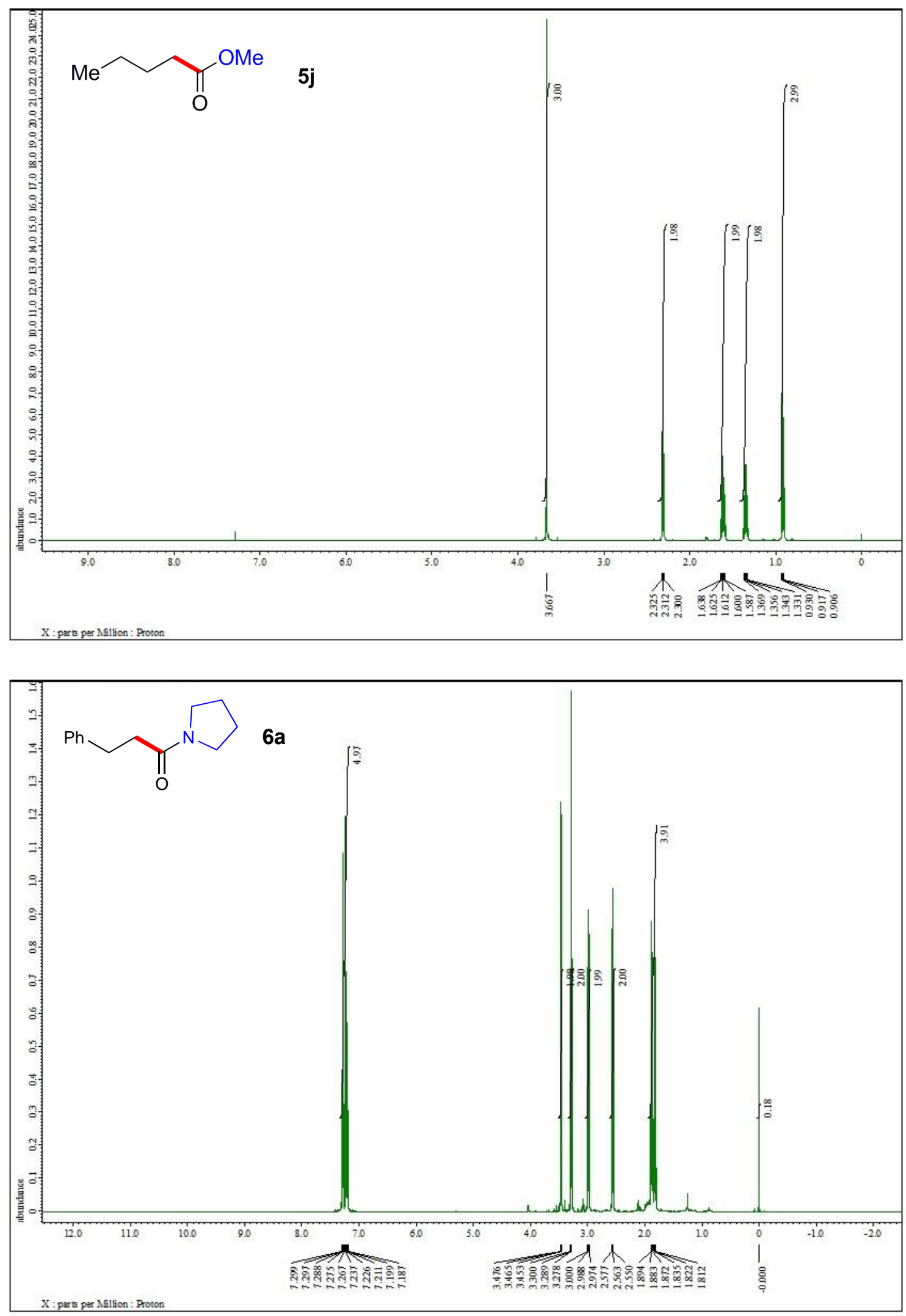

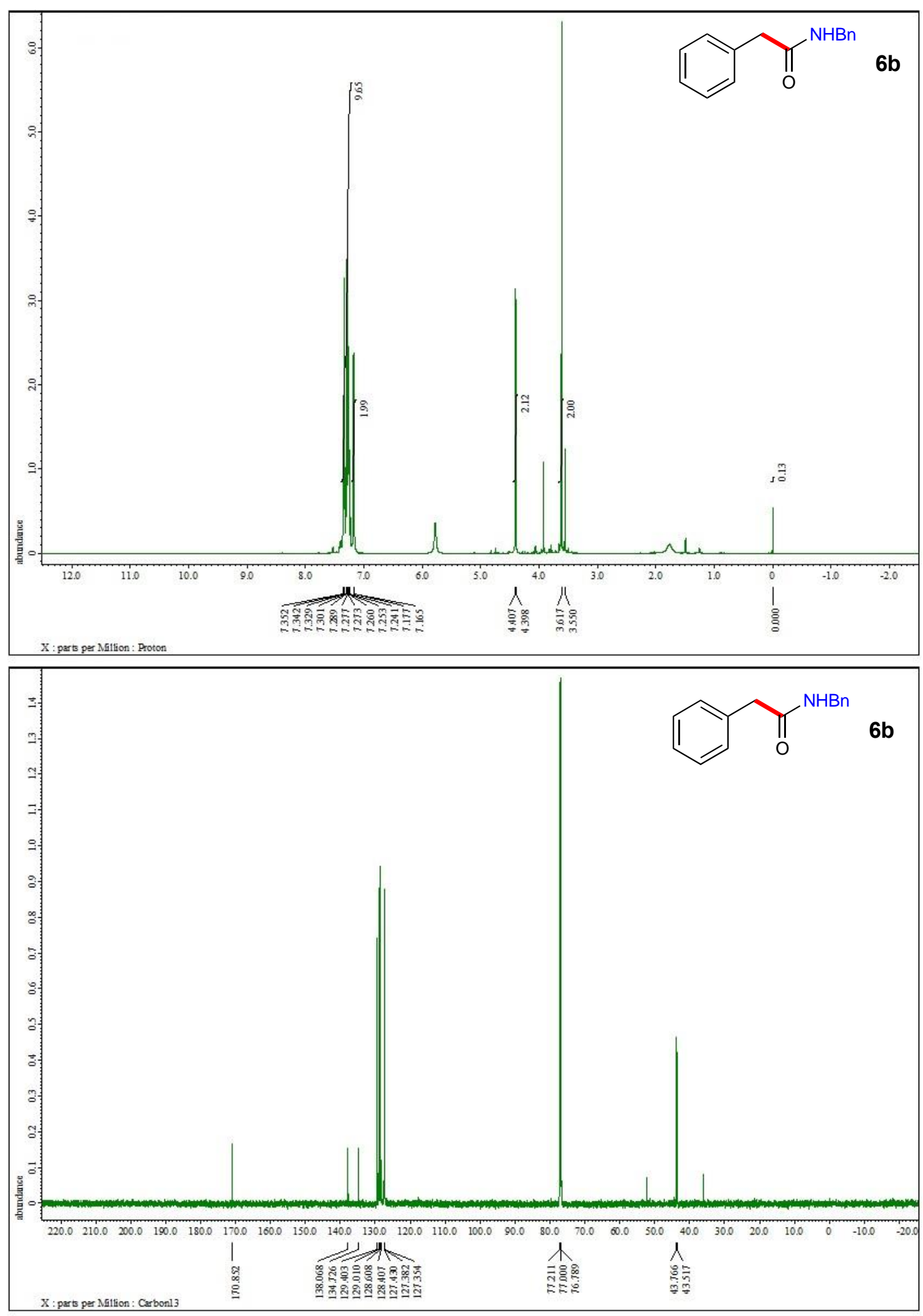

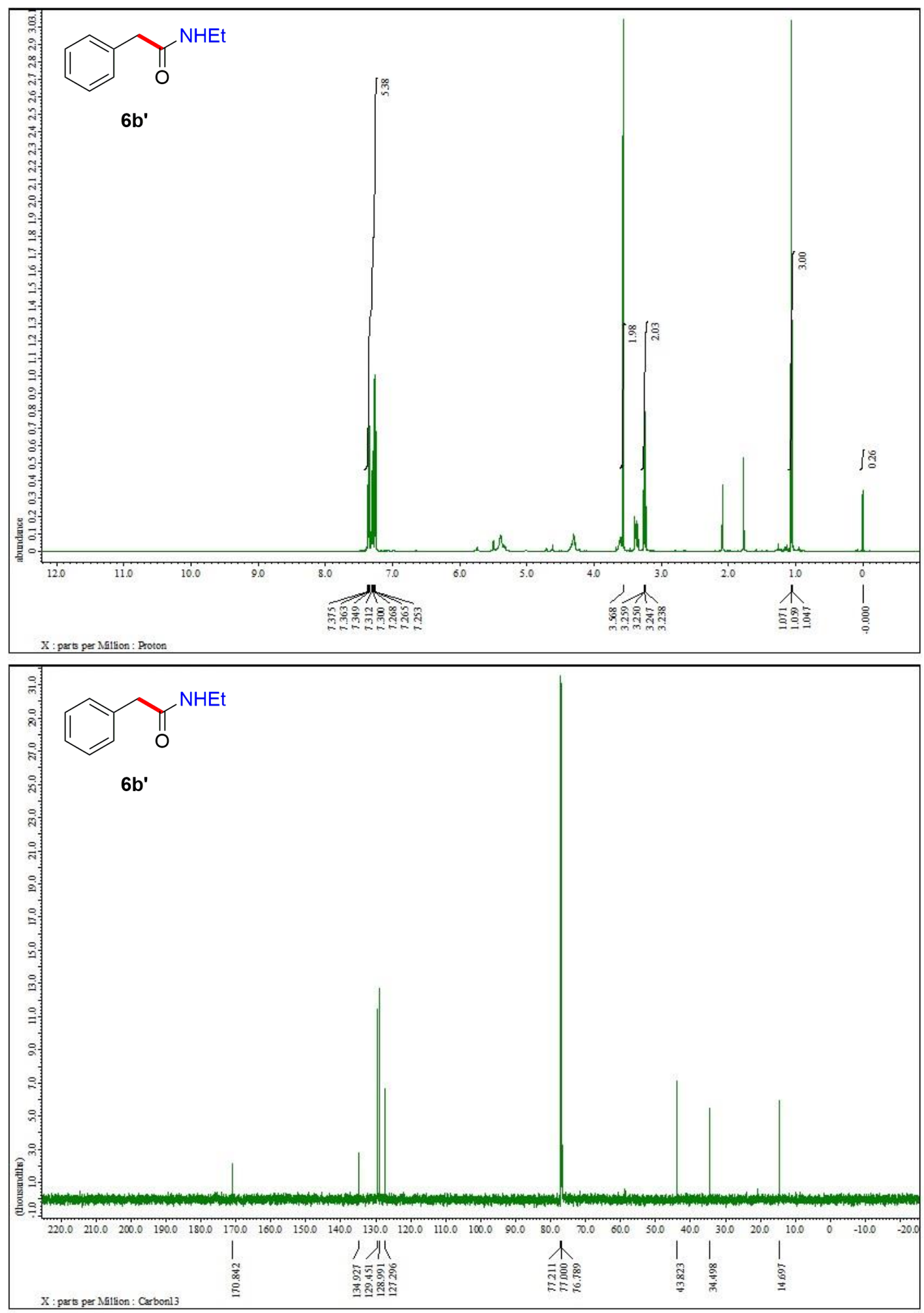

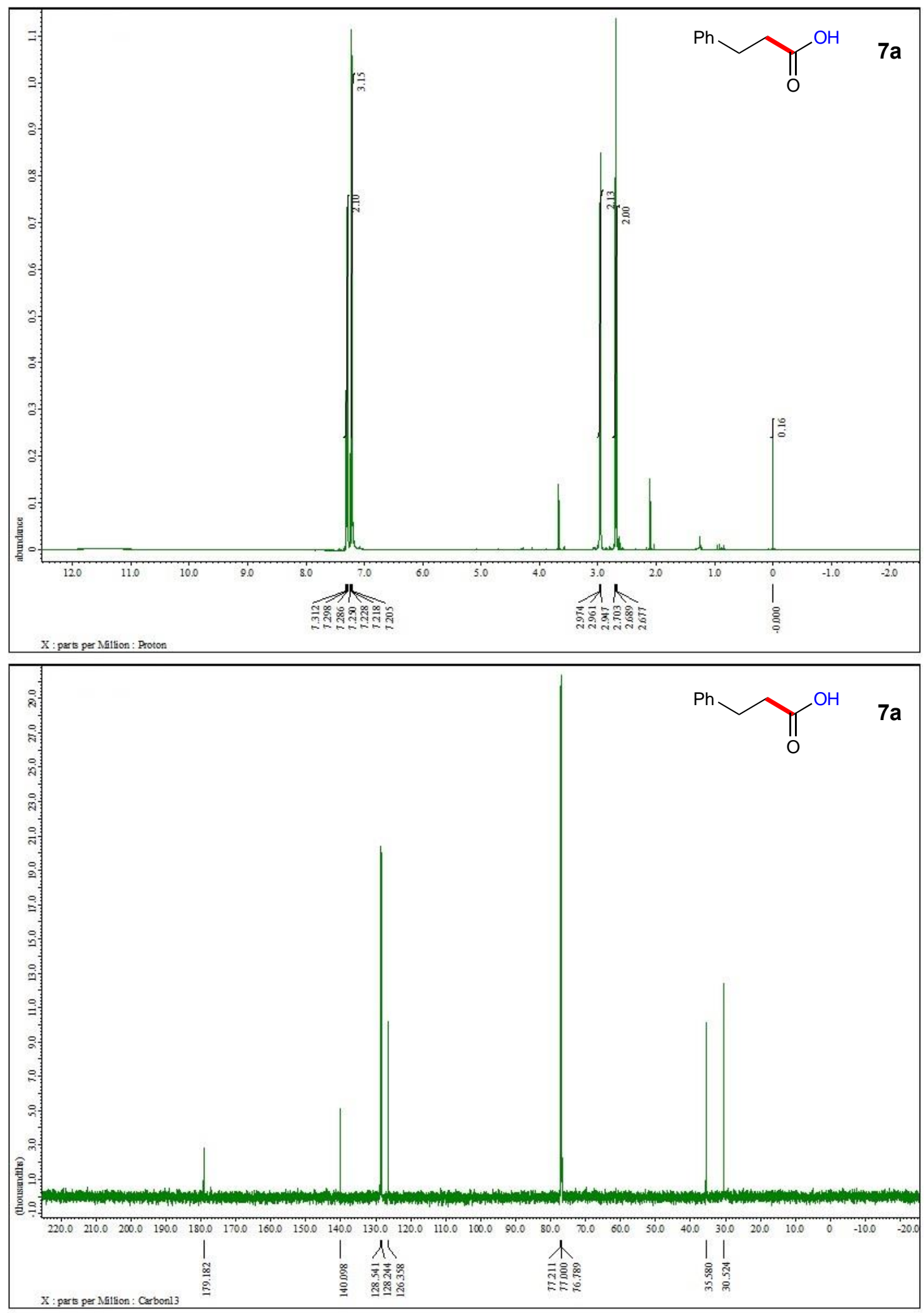

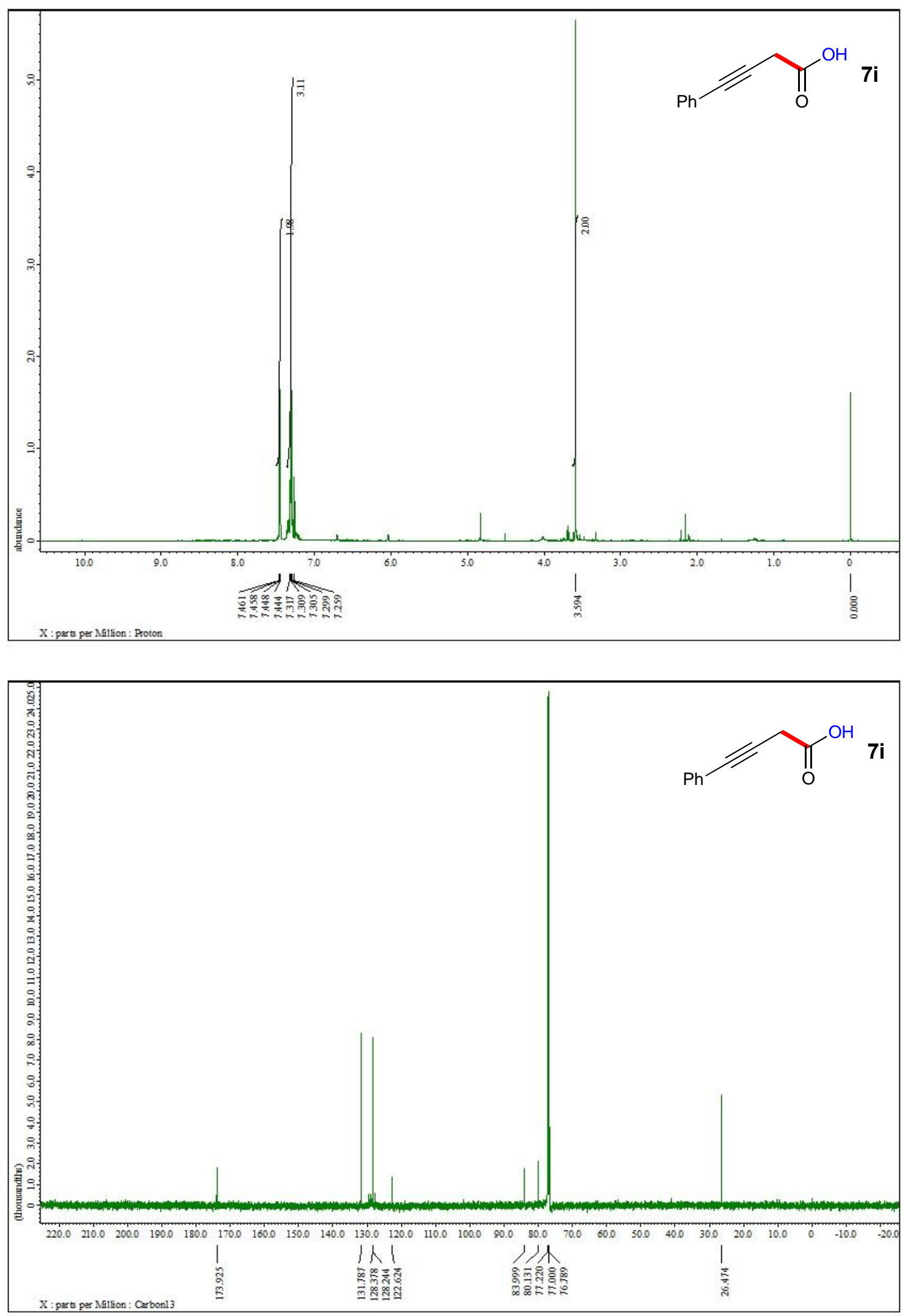